\title{
PERTINENCY OF HOPF ACTIONS AND QUOTIENT CATEGORIES OF COHEN-MACAULAY ALGEBRAS
}

\author{
Y.-H. BAO, J.-W. HE AND J. J. ZHANG
}

\begin{abstract}
We study invariants and quotient categories of fixed subrings of Artin-Schelter regular algebras under Hopf algebra actions.
\end{abstract}

\section{INTRODUCTION}

Artin-Schelter regular (or AS regular, for short) algebras [Definition 1.8 play an important role in noncommutative algebraic geometry. We study some classes of graded algebras that are closely related to AS regular algebras. In particular, we are interested in algebraic and homological properties of fixed subrings of AS regular algebras under semisimple Hopf algebra actions, or geometrically, properties of quotient singularities of noncommutative spaces under quantum group actions. Some homological properties of Hopf actions on AS regular algebras were studied in KKZ1. Finite dimensional Hopf actions on connected graded AS regular algebras of global dimension two with trivial homological determinant were classified in CKWZ1. In this paper we further investigate Hopf actions on AS regular algebras and the correspondence quotient singularities in the noncommutative setting.

One of most interesting features in this area is the McKay correspondence. The classical McKay correspondence connects many different topics in mathematics such as the finite subgroups of $\mathrm{SU}(2, \mathbb{C})$, Dynkin diagrams of $A D E$ type, simple Lie algebras, Kleinian singularities, $G$-Hilbert schemes and so on. See Bu, Le1, Le2] for very nice recent surveys and historical accounts. One ingredient in the classical or commutative McKay correspondence is the Auslander Theorem Au1, Au2 that states that, if $G$ is a finite small subgroup of $\mathrm{GL}\left(\mathbb{C}^{\oplus n}\right)$ acting on $R:=\mathbb{C}\left[x_{1}, \cdots, x_{n}\right]$ naturally, then there is a natural isomorphism of algebras

$$
R * G \cong \operatorname{End}_{R^{G}}(R) .
$$

Recall that a finite subgroup $G \subseteq \mathrm{GL}(V)$, for a finite dimensional vector space $V$, is called small if $G$ does not contain a pseudo-reflection of $V$.

Different extensions and noncommutative versions of the McKay correspondence have been proposed by several researchers Ch, CKWZ2, Mor2, Le2, Wem and many nice results have been proven. In noncommutative algebraic geometry, AS regular algebras are correct noncommutative analogues of the commutative polynomial rings. One key ingredient in noncommutative McKay correspondence is the Auslander Theorem when $R$ is replaced by a noncommutative AS regular algebra.

2000 Mathematics Subject Classification. Primary 16D90, 16E65, 16E10, Secondary 16B50, $16 \mathrm{E} 35$.

Key words and phrases. Artin-Schelter regular algebra, quotient category, Auslander theorem, Hopf algebra action, pertinency, Cohen-Macaulay property. 
It is desirable to prove the Auslander Theorem in a general noncommutative setting. Some partial results can be found in BHZ, CKWZ2, HVZ2, MU] and so on, under some extra hypotheses. One of the main goals of this paper is to prove a noncommutative version of the Auslander theorem that recovers most of the previous results and to provide some conjectures in this research direction.

Let $R$ be an $\mathbb{N}$-graded algebra over a base field $\mathbb{k}$. We are taking one step further by replacing the group $G$ in E0.0.1 by a Hopf algebra $H$. Let $H$ be a nontrivial semisimple Hopf algebra acting on $R$ homogeneously and inner faithfully [Definition 3.9. Let $R \# H$ denote the smash product [Mon, Definition 4.1.3]. Since $H$ is semisimple, the left and the right integrals of $H$ coincide. Denote by $\int$ the integral of $H$ such that $\varepsilon\left(\int\right)=1$. The main dimension function we are going to use here is the Gelfand-Kirillov dimension (also denoted by GK-dimension) - see KL or Definition 1.1. A new invariant we introduce in this paper is the following.

Definition 0.1. Retain the above notation and suppose that GKdim $R<\infty$. The pertinency of the $H$-action on $R$ is defined to be

$$
\mathrm{p}(R, H)=\operatorname{GKdim} R-\operatorname{GKdim}((R \# H) / I)
$$

where $I$ is the 2 -sided ideal of $R \# H$ generated by $1 \# \int$.

Fix an $R$ and an $H$, different $H$-actions on $R$ result in different pertinency. This means that $\mathrm{p}(R, H)$ is an invariant of the $H$-action on $R$, not just the pair $(R, H)$. When $H$ is the group algebra $\mathbb{k} G$, we write $\mathrm{p}(R, H)$ as $\mathrm{p}(R, G)$. If the $H$-action on $R$ is not inner faithful, then it is easy to check that $\mathrm{p}(R, H)=0$. If $H$ acts on a domain $R$ inner faithfully and $R \# H$ is prime, it is automatic that $\mathrm{p}(R, H) \geq 1$ (see Lemma 3.10). Note that $\mathrm{p}(R, H)=\operatorname{GKdim} R$ (or equivalently, $\operatorname{GKdim}((R \# H) / I)=0)$ if and only if $R / R^{H}$ is a right $H^{*}$-dense Galois extension in the sense of [HVZ2].

Definition 0.2. Let $R$ be a noetherian algebra with finite GK-dimension. Suppose $H$ is a Hopf algebra with integral $\int$ acting on $R$ inner faithfully, and let $M$ be a finitely generated right $R$-module.

(1) The grade of $M$ is defined to be

$$
j(M):=\min \left\{i \mid \operatorname{Ext}_{R}^{i}(M, R) \neq 0\right\} .
$$

If $\operatorname{Ext}_{R}^{i}\left(M, R_{R}\right)=0$ for all $i$, then we say $j(M)=\infty$.

(2) We say that the $H$-action on $R$ is homologically small if $j(R \# H /(I)) \geq 2$, where $I$ is the 2 -sided ideal generated by $1 \# \int$.

In part of the paper, we use Cohen-Macaulay property [Definition 1.4(3)] which is a homological property in the noncommutative setting that is intimately connected with the AS regularity. For example, all well-studied noetherian AS regular algebras have been proven to be Cohen-Macaulay and it is conjectured that the CohenMacaulay property is a consequence of the AS regularity.

Setup: For the rest of introduction, let $R$ denote a noetherian, connected graded $A S$ regular, Cohen-Macaulay algebra of GK-dimension $\geq 2$.

In the commutative (graded) case, homological smallness is equivalent to the (usual) smallness [BHZ, Lemma 7.2]. Our first result connects the noncommutative Auslander theorem with the pertinency.

Theorem 0.3. Let $H$ be a semisimple Hopf algebra acting on $R$ inner faithfully and homogeneously. Then the following are equivalent. 
(1) $\mathrm{p}(R, H) \geq 2$.

(2) The $H$-action on $R$ is homologically small.

(3) There is a natural isomorphism of algebras $R \# H \cong \operatorname{End}_{R^{H}}(R)$.

In Theorems 0.3 and 0.6 below, the Cohen-Macaulay property is crucial while the AS regularity is hidden in the background. Then, in Theorem 0.7 the AS regularity is definitely needed.

Theorem 0.3 does not recover the classical Auslander theorem, however, it extends most of previous results concerning the noncommutative Auslander theorem in CKWZ3, HVZ2, MU]. It is easier to manipulate the condition on $\mathrm{p}(R, H)$ than to prove the isomorphism in Theorem $0.3(3)$ directly, see $\mathrm{BHZ}$. This is one of the advantages of Theorem 0.3 .

By the above theorem, checking the hypothesis on the pertinency " $\mathrm{p}(R, H) \geq 2$ " becomes essential. In some cases, the inequality $\mathrm{p}(R, H) \geq 2$ is checkable - see Theorem 0.5 and $\mathrm{BHZ}$. We do not have a general algorithm of calculating this number. However, the following conjecture is expected.

Conjecture 0.4. Retain the hypotheses as in Theorem 0.3. Suppose the H-action on $R$ has trivial homological determinant in the sense of [KKZ1, Definition 3.3]. Then $\mathrm{p}(R, H) \geq 2$, or equivalently, the $H$-action on $R$ is homologically small.

When $R$ is the commutative polynomial ring and $H$ is a finite group algebra, this conjecture holds as a consequence of the classical Auslander theorem. In the noncommutative setting, this has been verified in some special cases, but the conjecture is open in general. If Conjecture 0.4 holds, then, by combining with Theorem 0.3 we have the following version of the Auslander theorem: Retain the hypotheses as in Theorem 0.3 and further assume that the $H$-action on $R$ has trivial homological determinant. Then $R \# H \cong \operatorname{End}_{R^{H}}(R)$ naturally.

Note that the aim of paper CKWZ2 is to establish a noncommutative McKay correspondence for Hopf algebra actions on AS regular algebras of dimension two, and one of the main results there is to verify Conjecture 0.4 when gldim $R=2$.

As a consequence of Theorem 0.3 , we have the following result. For any graded algebra $A$, let $\operatorname{Aut}_{g r}(A)$ denote the group of all graded algebra automorphisms of $A$. Suppose $n \geq 2$ and let $R:=\mathbb{k}_{-1}\left[x_{1}, \cdots, x_{n}\right]$ be the (-1)-skew polynomial ring generated by $x_{1}, \cdots, x_{n}$ and subject to the relations $x_{i} x_{j}=-x_{j} x_{i}$ for all $i \neq j$. Then $\operatorname{Aut}_{g r}(R)=\left(\mathbb{k}^{\times}\right)^{n} \rtimes S_{n}$ [KKZ2, Lemma 1.12(1)].

Theorem 0.5. Let $\sigma$ be the automorphism of $R:=\mathbb{k}_{-1}\left[x_{1}, \cdots, x_{n}\right]$ determined by sending $x_{i} \mapsto x_{i+1}$ for all $1 \leq i \leq n-1$ and $x_{n} \mapsto x_{1}$. Let $G$ be the group generated by $\sigma$. Then the homological determinant of the $G$-action on $R$ is trivial and $\mathrm{p}(R, G) \geq 2$. As a consequence, $R * G \cong \operatorname{End}_{R^{G}}(R)$.

Theorem 0.5 answers a question of Ellen Kirkman [Ki, see also Question 5.9. In contrast, when $R$ is a commutative polynomial ring $\mathbb{k}\left[x_{1}, \cdots, x_{n}\right]$ and $G$ is generated by the permutation sending $x_{i} \mapsto x_{i+1}$ for all $1 \leq i \leq n-1$ and $x_{n} \mapsto x_{1}$, then

$$
\mathrm{p}(R, G)= \begin{cases}1 & n=2 \\ \geq 2 & n>2 .\end{cases}
$$

So, in the commutative case, $G$ is not (homologically) small when $n=2$, see BHZ, Examples 7.3 and 7.4] for further comments. 
Going back to a general (graded) algebra $A$, we write $\operatorname{Gr} A$ for the category of right graded $A$-modules, and gr $A$ for the subcategory of finitely generated right graded $A$-modules. For any integer $n$, let $\operatorname{Gr}_{n} A$ (respectively, $\operatorname{gr}_{n} A$ ) be the subcategory of $\operatorname{Gr} A$ (respectively, gr $A$ ) consisting of objects with GK-dimension not larger than $n$. Define the following quotient categories

$$
\mathrm{QGr}_{n} B:=\frac{\operatorname{Gr} B}{\operatorname{Gr}_{n} B} \quad \text { and } \quad \operatorname{qgr}_{n} B:=\frac{\operatorname{gr} B}{\operatorname{gr}_{n} B}
$$

When $n=0$, the quotient category $\mathrm{QGr}_{0} B$ (respectively, $\operatorname{qgr}_{0} B$ ) is just QGr $B$ (respectively, qgr $B$ ) defined in noncommutative projective geometry AZ or Tails $B$ (respectively, tails $B$ ) used by other authors [MU], Ue, Mor1, Mor2].

Our first result relating the pertinency and the quotient category is the following.

Theorem 0.6. Retain the hypotheses as in Theorem 0.3. Let $\alpha$ be an integer $\leq \mathrm{p}(R, H)$ and assume $\mathrm{GK} \operatorname{dim} R=n$. Then there is an equivalence of categories

$$
\operatorname{qgr}_{n-\alpha} R^{H} \cong \operatorname{qgr}_{n-\alpha} R \# H \text {. }
$$

Since $R$ is AS regular, $R \# H$ has finite global dimension. If, in addition, $R$ is PI (namely, $R$ satisfies a polynomial identity), then the quotient category $\operatorname{qgr}_{n-\alpha} R \# H$ has finite global dimension. Under the extra PI hypothesis, by the above theorem, $\operatorname{qgr}_{n-\alpha} R^{H}$ has finite global dimension. When a connected graded algebra $A$ is PI, it is reasonable to define the dimension of the singular locus of $A$ to be

$$
\operatorname{dim}_{\text {sing }}(A)=\min \left\{i \mid \operatorname{qgr}_{i} A \text { has finite global dimension }\right\} .
$$

Using this definition and assuming $R$ is PI, we can bound the $\operatorname{dim}_{\text {sing }}$ of the fixed subring $R^{H}$ by

$$
\operatorname{dim}_{\text {sing }}\left(R^{H}\right) \leq n-\mathrm{p}(R, H)=\operatorname{gldim} R-\mathrm{p}(R, H) .
$$

In particular, when $\mathrm{p}(R, H)=\operatorname{gldim} R, R^{H}$ has graded isolated singularities in the sense of Ueyama. Recall from [Ue, Definition 2.2] a noetherian connected graded algebra $A$ is a graded isolated singularity if tails $A(=\operatorname{qgr} A$ ) has finite global dimension. We give new examples of noncommutative graded isolated singularities in Proposition 5.4. Following the idea of the noncommutative crepant resolutions Le2, $R \# H$ should be viewed a resolution of singularities of $R^{H}$. Some ideas in this paper are motivated by recent papers CKWZ1, CKWZ2, Le2, Mor1, Mor2, MU, HVZ2, Ue].

Another result concerning quotient categories is a version of BGG correspondence. Let $R$ be a Koszul AS regular algebra and $E$ the graded algebra

$$
E:=\bigoplus_{i=0}^{n} \operatorname{Ext}_{R \# H}^{i}(S, S)
$$

where $S$ is a direct sum of all non-isomorphic graded simple right modules over $R \# H$ concentrated in degree zero. Since $R$ is Koszul, so is $R \# H$. As a consequence, $E$ is Koszul. (All these are $\mathbb{N}$-graded and locally finite.) Since $R$ is AS regular, $E$ is Frobenius. Then the stable module category gr $E$ is a triangulated category. Let $M$ be a finite dimensional right graded $E$-module. Take a minimal graded projective resolution of $M$ as follows

$$
\cdots \longrightarrow P^{-n} \longrightarrow \cdots \longrightarrow P^{0} \longrightarrow M \longrightarrow 0 .
$$


The complexity of $M$, denoted by $c(M)$, is the least integer $\lambda \geq 0$ such that $\operatorname{dim} P^{-n}<a n^{\lambda-1}$ for almost all $n$, where $a>0$ is a fixed number. The complexity of a module over a finite dimension algebra is an important invariant in studying the representations of finite dimensional algebras [Ca, CDW, GLW]. Given any nonnegative integer $\beta$, let $\mathcal{C}_{\beta}$ be the full subcategory of gr $E$ consisting of objects $M$ with $c(M) \leq \beta$.

Theorem 0.7. Retain the hypotheses as in Theorem 0.3 and assume further that $R$ is Koszul and that $\mathbb{k}$ is algebraically closed. Let $\beta$ be a nonnegative integer.

(1) There is a canonical anti-equivalences of triangulated categories

$$
D^{b}\left(\operatorname{qgr}_{\beta} R \# H\right) \cong \underline{\operatorname{gr}} E / \mathcal{C}_{\beta}
$$

(2) For any $\beta \geq \mathrm{GKdim} R-\mathrm{p}(R, H)$, there is an canonical anti-equivalence of triangulated categories

$$
D^{b}\left(\operatorname{qgr}_{\beta} R^{H}\right) \cong \underline{\operatorname{gr}} E / \mathcal{C}_{\beta} .
$$

When $H$ is a group algebra, one can define another invariant associated to the $G$-action on $R$. Let now $R$ be as in Theorem 0.7 Then the global dimension and GK-dimension of $R$ are the same. For any graded algebra automorphism $g$ of $R$, one can define the trace function $\operatorname{Tr}(g, t)=: \sum_{i=0}^{\infty} \operatorname{Tr}\left(\left.g\right|_{R_{i}}\right) t^{i}$ as in $\underline{\mathrm{KKZ2}}$, Section $1]$.

Definition 0.8. Let $g$ be a graded algebra automorphism of $R$ of finite order and let $G$ be a finite subgroup of $\operatorname{Aut}_{g r}(R)$.

(1) The reflection number of $g$ is defined to be

$$
\mathrm{r}(g)=\operatorname{GKdim} R-\text { the order of the pole of } \operatorname{Tr}(g, t) \text { at } t=1 .
$$

(2) [KKZ3, Definition 2.2] $g$ is called a quasi-reflection if $r(g)=1$.

(3) [KKZ4, Definition 3.7(b)] $g$ is called a quasi-bireflection if $r(g)=2$.

(4) The reflection number of $G$-action on $R$ is defined to be

$$
\mathrm{r}(R, G)=\min \{\mathrm{r}(g) \mid 1 \neq g \in G\} .
$$

(5) $G$ is called conventionally small if $\mathrm{r}(R, G) \geq 2$, or equivalently, $G$ does not contain any quasi-reflection.

Similar to $\mathrm{p}(R, G), \mathrm{r}(R, G)$ is an invariant of the $G$-action on $R$, not of the pair $(R, G)$. The relation between $\mathrm{p}(R, G)$ and $\mathrm{r}(R, G)$ is not straightforward, and what we are hoping for is the following conjectural inequality.

Conjecture 0.9. Retain the hypotheses as in Theorem 0.7 . Let $G$ be a finite subgroup of $\operatorname{Aut}_{g r}(R)$. Then

$$
\mathrm{p}(R, G) \geq \mathrm{r}(R, G) .
$$

This conjecture can be verified for some special cases [Theorem [5.7(4)]. If it holds, then Conjecture 0.4 follows when $H$ is a group algebra because $r(R, G) \geq$ 2 when the $G$-action has trivial homological determinant. Indeed, if $g \in G$ is a quasi-reflection, then $\operatorname{Tr}(g, t)=\frac{1}{(1-t)^{n-1}(1-\xi t)}$ where $n=\operatorname{GKdim} R$ and $\xi \neq$ 1. Since the homological determinant of $G$-action is trivial, we obtain that $\xi=$ 1 , a contradiction. Therefore $G$ contains no quasi-reflections, that is, $\mathrm{r}(R, G) \geq$ 2. If Conjecture 0.9 holds, then, by combining with Theorem 0.3 , we obtain the 
following: Retain the hypotheses as in Theorem 0.7 and further assume that the $G$ is conventionally small. Then $R * G \cong \operatorname{End}_{R^{G}}(R)$ naturally.

Combining (E0.6.1) with E0.8.1 we have another conjecture

$$
\operatorname{dim}_{\text {sing }}\left(R^{G}\right) \leq \operatorname{gldim} R-\mathrm{r}(R, G) .
$$

The advantage of this conjectural inequality is that, in contrast to $\mathrm{p}(R, G)$, the invariant $\mathrm{r}(R, G)$ is relatively easy to calculate. Note that E0.8.1 can be verified when $R$ is AS regular of global dimension two following the classification of all such $G$-actions in CKWZ1. When the $G$-action on $R$ has trivial homological determinant, then $\mathrm{p}(R, G)$ (respectively, $\mathrm{r}(R, G)$ ) would be either 2 or 3 and $\operatorname{dim}_{\text {sing }}\left(R^{G}\right)$ would be either 0 or 1 . Understanding all possibilities of $\left\{\mathrm{p}(R, G), \mathrm{r}(R, G), \operatorname{dim}_{\text {sing }}\left(R^{G}\right)\right\}$ in dimension three should be an interesting project.

This paper is organized as follows. We provide background material in Section 1. In Section 2, we prove a Morita type theorem for the equivalences of the quotient categories. In Section 3, we consider semisimple Hopf actions on graded CohenMacaulay algebras. We prove a generalized version of Theorem 0.3 and then give a proof of Theorem 0.6. In Section 4, we establish a version of BGG correspondence between the derived categories of quotient categories and the stable categories of

some Frobenius categories. In particular, we obtain Theorem 0.7 In Section 5, we compute the pertinency of finite group actions on some special algebras, and give a proof of Theorem 0.5 .

\section{Preliminaries}

Throughout let $\mathbb{k}$ be a base ring that is a noetherian commutative domain. All algebraic objects are over $\mathbb{k}$. An algebra means an associative $\mathbb{k}$-algebra that is projective as a $\mathbb{k}$-module. In Sections 4 and 5 (and part of Section 3) we further assume that $\mathbb{k}$ is a field. Let $B$ be a (left and right) noetherian algebra. Usually we are working with right $B$-modules. We write $\operatorname{Mod} B$ for the category of all right $B$-modules, and $\bmod B$ for the full subcategory of all finitely generated (or f.g. for short) right $B$-modules.

Several results in this paper concern quotient categories of the module categories defined via a dimension function. For the purpose of this paper, a function

$$
\partial: \operatorname{Mod} B \longrightarrow \mathbb{R} \cup\{ \pm \infty\}
$$

is called a dimension function if, for all f.g. B-modules $M$,

$$
\partial(M) \geq \max \{\partial(N), \partial(M / N)\},
$$

whenever $N$ is a submodule of $M$. The $\partial$ is called an exact dimension function if for all f.g. $B$-modules $M$,

$$
\partial(M)=\max \{\partial(N), \partial(M / N)\}
$$

whenever $N$ is a submodule of $M$. If $M$ is not f.g., we extend the definition of $\partial$ by

$$
\partial(M)=\sup \{\partial(N) \mid \text { for all f.g. } B \text {-submodules } N \subseteq M\} .
$$

By using (E1.0.3), equation (E1.0.2) holds for all $B$-modules $M$ as well. Our definition of a dimension function is weaker than the definition given in [MR, 6.8.4], however, E1.0.2 is the only property we will use.

For a $\mathbb{k}$-module $V, \operatorname{dim} V$ denotes $\operatorname{dim}_{Q}\left(V \otimes_{\mathbb{k}} Q\right)$ where $Q$ is the fraction field of $\mathbb{k}$. One special dimension function is the following 
Definition 1.1. KL, Definition 2.1] Let $B$ be an algebra and $M$ a right $B$-module.

(1) The Gelfand-Kirillov dimension or GK-dimension of $B$ is defined to be

$\operatorname{GKdim} B=\sup _{V}\left\{\varlimsup_{n \rightarrow \infty} \log _{n}\left(\operatorname{dim} V^{n}\right) \mid\right.$ f.g. $\mathbb{k}$-submodules $\left.V \subseteq B\right\}$.

(2) The Gelfand-Kirillov dimension or GK-dimension of $M$ is defined to be

$\operatorname{GKdim} M=\sup _{V, W}\left\{\varlimsup_{n \rightarrow \infty} \log _{n}\left(\operatorname{dim} W V^{n}\right) \mid\right.$ f.g. $\mathbb{k}$-submodules $\left.V \subseteq B, W \subseteq M\right\}$.

See $\mathrm{KL}$ and Remark 1.5 for basic properties of the GK-dimension.

In a major part of the paper the dimension function is chosen to be the GelfandKirillov dimension. However, a general dimension function will be useful when we deal with the local setting in the sequel $\mathrm{BHZ}$ in which case the GK-dimension is usually infinite and does not work well.

From now on we fix an exact dimension function $\partial$. Given an integer $n \geq 0$, let $\operatorname{Mod}_{n} B$ denote the full subcategory of $\operatorname{Mod} B$ consisting of right $B$-modules with $\partial$-dimension less than or equal to $n$, and let

$$
\bmod _{n} B=\operatorname{Mod}_{n} B \bigcap \bmod B .
$$

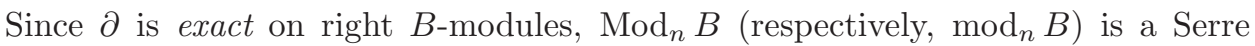
subcategory of $\operatorname{Mod} B($ respectively, $\bmod B)$. Hence it makes sense to define the quotient categories:

$$
\operatorname{QMod}_{n} B:=\frac{\operatorname{Mod} B}{\operatorname{Mod}_{n} B}, \quad \text { and } \operatorname{qmod}_{n} B:=\frac{\bmod B}{\bmod _{n} B} .
$$

We denote the natural projection functor by

$$
\pi: \operatorname{Mod} B \longrightarrow \operatorname{QMod}_{n} B \text {. }
$$

For $M \in \operatorname{Mod} B$, we will write $\mathcal{M}$ for the object $\pi(M)$ in $\operatorname{QMod}_{n} B$. The hom-set of the objects in the quotient category is defined by

$$
\operatorname{Hom}_{\mathrm{QMod}_{n} B}(\mathcal{M}, \mathcal{N})=\underset{\longrightarrow}{\longrightarrow} \operatorname{Hom}_{B}\left(M^{\prime}, N^{\prime}\right)
$$

for $M, N \in \operatorname{Mod} B$, where $M^{\prime}$ is a submodule of $M$ such that $\partial\left(M / M^{\prime}\right) \leq n$, $N^{\prime}=N / T$ for some submodule $T$ with $\partial(T) \leq n$, and the direct limit runs over all the pairs $\left(M^{\prime}, N^{\prime}\right)$ with these properties.

Definition 1.2. Let $A$ and $B$ be noetherian algebras and $\partial$ be an exact dimension function that is defined on right $A$-modules and on right $B$-modules. Let $n$ and $i$ be nonnegative integers. Let ${ }_{A} M_{B}$ denote one (or any) bimodule which is f.g. both as a left $A$-module and as a right $B$-module.

(1) We say $\partial$ satisfies $\gamma_{n, i}(M)$ if for any $N \in \bmod _{n} A$, $\operatorname{Tor}_{j}^{A}(N, M) \in \bmod _{n} B$ for all $0 \leq j \leq i$.

(2) We say $\partial$ satisfies $\gamma_{n, i}$ if it satisfies $\gamma_{n, i}(M)$ for all $M$ given as above.

In the next section we will be particularly interested in the $\gamma_{n, 1}$ property.

Lemma 1.3. Let $A$ and $B$ be noetherian algebras such that $\partial$ is an exact dimension function on both right $A$-modules and right $B$-modules. Assume that a bimodule ${ }_{A} M_{B}$ is f.g. as a left $A$ and as a right $B$-module and that $\partial$ satisfies $\gamma_{n, 1}(M)$.

(1) The functor $-\otimes_{A} M$ induces a functor

$$
-\otimes_{\mathcal{A}} \mathcal{M}: \operatorname{qmod}_{n} A \longrightarrow \operatorname{qmod}_{n} B \text {. }
$$


(2) The functor $-\otimes_{A} M$ induces a functor

$$
-\otimes_{\mathcal{A}} \mathcal{M}: \mathrm{QMod}_{n} A \longrightarrow \mathrm{QMod}_{n} B .
$$

Proof. We first prove (2).

For $K \in \operatorname{Mod}_{n} A$, it is a direct limit of f.g. submodules. Since the direct limit is an exact functor and, by assumption, $\partial$ is exact and satisfies $\gamma_{n, 1}(M)$, we obtain that $\partial\left(K \otimes_{A} M\right) \leq n$ and that $\partial\left(\operatorname{Tor}_{1}^{A}(K, M)\right) \leq n$ for all $K \in \operatorname{Mod}_{n} A$. Let $f: U \rightarrow V$ be a morphism in $\operatorname{Mod} A$ such that $\operatorname{ker} f$ and coker $f$ are in $\operatorname{Mod}_{n} B$. By the right exactness of $-\otimes_{A} M$, the cokernel of the right $B$-module morphism

$$
f \otimes_{A} M: U \otimes_{A} M \rightarrow V \otimes_{A} M
$$

is in $\operatorname{Mod}_{n} B$. Write $f$ as the composition

$$
f=h \circ g: U \stackrel{g}{\rightarrow} W \stackrel{h}{\hookrightarrow} V .
$$

Since $\partial\left(\operatorname{Tor}_{1}^{A}(\operatorname{coker}(f), M)\right) \leq n$, by the long exact sequence associated to $-\otimes_{A} M$, $\partial\left(\operatorname{ker}\left(h \otimes_{A} M\right)\right) \leq n$. Let $X=\operatorname{ker} f=\operatorname{ker} g$. Then $\partial(X) \leq n$. We have an exact sequence

$$
X \otimes_{A} M \longrightarrow U \otimes_{A} M \stackrel{g \otimes_{A} M}{\longrightarrow} W \otimes_{A} M \longrightarrow 0
$$

which induces the exact sequence

$$
X \otimes_{A} M \longrightarrow \operatorname{ker}\left(f \otimes_{A} M\right) \longrightarrow \operatorname{ker}\left(h \otimes_{A} M\right) \longrightarrow 0 .
$$

Since $\partial$ is exact, we see

$$
\partial\left(\operatorname{ker}\left(f \otimes_{A} M\right)\right) \leq \max \left\{\partial\left(X \otimes_{A} M\right), \partial\left(\operatorname{ker}\left(h \otimes_{A} M\right)\right)\right\} \leq n .
$$

Hence $f \otimes_{A} M$ has its kernel and cokernel in $\operatorname{Mod}_{n} B$. Therefore, $-\otimes_{A} M$ induces a functor

$$
-\otimes_{\mathcal{A}} \mathcal{M}: \mathrm{QMod}_{n} A \longrightarrow \mathrm{QMod}_{n} B .
$$

So we have proven part (2).

Since $M$ is f.g. in both sides, this functor restricts to

$$
-\otimes_{\mathcal{A}} \mathcal{M}: \operatorname{qmod}_{n} A \longrightarrow \operatorname{qmod}_{n} B .
$$

Therefore (1) follows.

We now recall a definition.

Definition 1.4. Let $B$ be an algebra and $M$ a right $B$-module.

(1) ASZ1, Definition 0.4] Let $\partial$ be a dimension function. We say $B$ is $\partial$-CohenMacaulay (or $\partial-C M$ ) if $\partial(B)=d \in \mathbb{N}$, and

$$
j(M)+\partial(M)=\partial(B),
$$

for every f.g. right $B$-module $M \neq 0$.

(2) If $B$ is GKdim-Cohen-Macaulay, we just say it is Cohen-Macaulay or CM.

(3) Let $\partial$ be an exact dimension function and $\partial(B)=d \in \mathbb{N}$. Let $s$ be a nonnegative integer. We say $B$ is $\partial-\mathrm{CM}(s)$ if, for every f.g right $B$-module $M$,

$$
\partial(M) \leq d-s \Longleftrightarrow j(M) \geq s .
$$

We just say $B$ is $\operatorname{CM}(s)$ if it is $\operatorname{GKdim-CM}(s)$.

The CM property together with the Artin-Schelter regularity and the Auslander property (another homological property) has been studied in the noncommutative setting in ASZ1, ASZ2, SZ, YZ, Zh. 
Remark 1.5. In parts $(4,5)$, assume that $\mathbb{k}$ is a field.

(1) The dimension function $\partial$ is exact on $\partial-\mathrm{CM}$ algebras ASZ2, p. 3].

(2) GKdim is exact on filtered algebras such that the associated graded algebras are locally finite and noetherian $\mathrm{KL}$, Theorem 6.14].

(3) GKdim satisfies $\gamma_{n, 0}$ for all $n$. Namely, for any $N \in \bmod _{n} A$, we have GKdim $N \otimes_{A} M \leq n$, assuming ${ }_{A} M_{B}$ is f.g. in both sides. This follows from [KL, Proposition 5.6] by a slight modification of the proof.

(4) For every f.g. module $N$, it is well-known that $\operatorname{GKdim} N=0$ if and only if $N$ is finite dimensional over $\mathbb{k}$. Using this fact, one can easily check that $\operatorname{GKdim} \operatorname{Tor}_{i}^{A}(N, M)=0$ if GKdim $N=0$ and ${ }_{A} M_{B}$ is f.g. in both sides. This means that GKdim satisfies $\gamma_{0, i}$ for all $i$.

(5) Take $\partial=$ GKdim. As a consequence of part (4) and Lemma 1.3(2), the functor

$$
-\otimes_{\mathcal{A}} \mathcal{M}: \mathrm{QMod}_{0} A \longrightarrow \mathrm{QMod}_{0} B
$$

is always well-defined.

Let $B=\bigoplus_{n>0} B_{n}$ be a noetherian graded algebra. In this paper, a graded algebra means an $\mathbb{N}$-graded and a graded module means $\mathbb{Z}$-graded. We call $B$ locally finite if each $B_{n}$ has finite rank over $\mathbb{k}$. We write $\operatorname{Gr} B$ for the category of right graded $B$-modules, and $\operatorname{gr} B$ for the subcategory of f.g. right graded $B$ modules. Let $\partial$ be an exact dimension function on graded right $B$-modules. Similar to the nongraded case, we write $\mathrm{Gr}_{n} B$ (respectively, $\operatorname{gr}_{n} B$ ) for the subcategory of $\operatorname{Gr} B$ (respectively, gr $B$ ) consisting of objects with $\partial$-dimension not larger than $n$. Similar to the definitions given in the introduction, we have quotient categories

$$
\mathrm{QGr}_{n} B:=\frac{\mathrm{Gr} B}{\operatorname{Gr}_{n} B} \quad \text { and } \quad \operatorname{qgr}_{n} B:=\frac{\operatorname{gr} B}{\operatorname{gr}_{n} B} .
$$

We also use $\pi: \operatorname{Gr} B \rightarrow \mathrm{QGr}_{n} B$ for the natural projection functor, and for $M \in$ Gr $B$, we write $\mathcal{M}:=\pi(M)$.

If $M$ is a f.g. graded right module over a noetherian locally finite graded algebra $B$, then its GK-dimension can be computed by [Zh, (E7)]

$$
\operatorname{GKdim} M=\varlimsup_{k \rightarrow \infty} \log _{k} \sum_{j \leq k} \operatorname{dim}\left(M_{j}\right) .
$$

Let $M$ and $N$ be right $A$ modules. As usual, we write

$$
\underline{\operatorname{Hom}}_{A}(M, N)=\oplus_{j \in \mathbb{Z}} \operatorname{Hom}_{\mathrm{Gr}} A(M, N(j)),
$$

where $\operatorname{Hom}_{\operatorname{Gr}} A(M, N)$ is the space of all the graded $A$-module homomorphisms preserving the gradings. If $N$ is a graded $A$ - $B$-bimodule, the tensor product $M \otimes_{A} N$ is a right graded $B$-module by setting the $j$ th component $\left(M \otimes_{A} N\right)_{j}$ to be the subspace of $M \otimes_{A} N$ spanned by elements $x \otimes_{A} y$, where $x \in M_{k}$ and $y \in N_{j-k}$ for all $k \in \mathbb{Z}$. The $i$ th derived functor of the $\underline{\operatorname{Hom}}_{A}(M, N)$ in the graded category (respectively, $M \otimes_{A} N$ ) is denoted by $\underline{\operatorname{Ext}}_{A}^{i}(M, N)$ (respectively, $\underline{\operatorname{Tor}}_{i}^{A}(M, N)$ ).

Lemma 1.6. Let $A$ and $B$ be noetherian locally finite graded algebras. Then GKdim on graded modules satisfies $\gamma_{n, i}$ for all $n$ and $i$.

Proof. Let $M$ be a graded $A$-B-bimodule such that $M$ is f.g. on both sides. We need to show that, for every f.g. graded right $A$-module $N$ with $\operatorname{GKdim} N \leq n$, we have $\operatorname{GKdim} \underline{\operatorname{Tor}}_{i}^{A}(N, M) \leq n$ for all $i \geq 0$. 
Since $N$ is f.g., $\underline{\operatorname{Tor}}_{i}^{A}(N, M)$ is a graded f.g. $B$-module for each $i \geq 0$. Let

$$
\cdots \longrightarrow F_{n} \longrightarrow \cdots \longrightarrow F_{1} \longrightarrow F_{0} \longrightarrow M \longrightarrow 0
$$

be a graded free resolution of the left graded $A$-module $M$. Since ${ }_{A} M$ is f.g., we can choose each $F_{i}$ to be f.g.. By definition, $\operatorname{Tor}_{i}^{A}(N, M)$ (forgetting the right $B$-module structure) is a subquotient of $N \otimes_{A} F_{i}$. Temporarily write $T:=\underline{\operatorname{Tor}}_{i}^{A}(N, M)$ and $\bar{N}:=N \otimes_{A} F_{i}$. Since $T$ is f.g. as a right graded $B$-module and, as a graded $\mathbb{k}$-module, $T$ is a subquotient of $\bar{N}$, by (E1.5.1), we have

$$
\begin{aligned}
\operatorname{GKdim} T & =\varlimsup_{k \rightarrow \infty} \log _{k} \sum_{j \leq k} \operatorname{dim}\left(T_{j}\right) \\
& \leq \varlimsup_{k \rightarrow \infty} \log _{k} \sum_{j \leq k} \operatorname{dim}\left(\bar{N}_{j}\right) \\
& \leq \varlimsup_{k \rightarrow \infty} \log _{k} \sum_{j \leq k} \operatorname{dim}\left(N_{j}\right) \\
& \leq \operatorname{GKdim} N=n .
\end{aligned}
$$

Lemma 1.7. Let $A$ and $B$ be noetherian locally finite graded algebras. Consider $\partial:=$ GKdim. For any graded $A$-B-bimodule $M$ that is f.g. on both sides, a graded version of Lemma 1.3 holds. Namely, for any $n \geq 0$, we have functors:

$$
-\otimes_{\mathcal{A}} \mathcal{M}: \mathrm{QGr}_{n} A \longrightarrow \mathrm{QGr}_{n} B, \quad \text { and } \quad-\otimes_{\mathcal{A}} \mathcal{M}: \operatorname{qgr}_{n} A \longrightarrow \operatorname{qgr}_{n} B .
$$

Proof. By Lemma 1.6. $\partial$ satisfies $\gamma_{n, i}(M)$ for all $n$ and $i$ and all $M$. The assertion follows from a graded version of the proof of Lemma 1.3 .

To conclude this section we recall and introduce a couple of more definitions.

The notion of Artin-Schelter Gorensteinness was generalized to the nonconnected graded case by several authors. We are going to use the following version. Let $A$ be a locally finite graded algebra such that $A_{0}$ is semisimple.

Definition 1.8. [MM, Definition 2.1] Let $\mathbb{k}$ be a field. A locally finite graded algebra $A$ with $A_{0}$ being semisimple is called Artin-Schelter Gorenstein (or AS Gorenstein) if the following conditions hold:

(a) $A$ has finite injective dimension $d$ on both sides,

(b) $\underline{\operatorname{Ext}}_{A}^{i}\left(A_{0}, A\right)=\underline{\operatorname{Ext}}_{A \text { op }}^{i}\left(A_{0}, A\right)=0$ for all $i \neq d$ where $A_{0}=A / A_{\geq 1}$, and

(c) $\underline{\operatorname{Ext}}_{A}^{d}\left(A_{0}, A\right) \cong A_{0}(l)$ and $\underline{\operatorname{Ext}}_{A^{\circ p}}^{d}\left(A_{0}, A\right) \cong A_{0}(l)$ for some integer $l$.

If moreover

(d) $A$ has finite global dimension, and

(e) $A$ has finite GK-dimension,

then $A$ is called Artin-Schelter regular (or $A S$ regular).

In [MM, Definition 2.1], the AS-Gorensteinness only requires that $A_{0}$ is finite dimensional and $\underline{\operatorname{Ext}}_{A}^{d}\left(A_{0}, A\right) \cong A_{0}^{*}(l)$ where $A_{0}^{*}=\operatorname{Hom}_{\mathbb{k}}\left(A_{0}, \mathbb{k}\right)$. When $A_{0}$ is semisimple, the two definitions coincides since $A_{0} \cong A_{0}^{*}$ as $A_{0}$-bimodules in this case.

In the introduction, $R$ is connected graded AS regular.

When $A$ is noetherian, connected graded and PI of finite global dimension, then $A$ is AS regular and CM [SZ, Theorem 1.1]. It is conjectured that every noetherian connected graded AS regular algebra is CM. 
Definition 1.9. Let $R$ be a subalgebra of $B$ and let $\partial$ be a dimension function defined on right $R$-modules and right $B$-modules.

(1) We say $\partial$ is weakly $B / R$-hereditary if $\partial\left(M_{R}\right) \leq \partial\left(M_{B}\right)$ for every f.g. right $B$-module $M$.

(2) Suppose $B_{R}$ is f.g.. We say $\partial$ is $B / R$-hereditary if for every f.g. right $B$-module $M, \partial\left(M_{R}\right)=\partial\left(M_{B}\right)$.

Lemma 1.10. Let $R$ be a subalgebra of $B$.

(1) GKdim is always weakly $B / R$-hereditary.

(2) Suppose that $R \subseteq B$ are noetherian and locally finite graded algebras such that $B_{R}$ is f.g. Then GKdim is $B / R$-hereditary on graded right modules.

Proof. (1) This follows from Definition 1.1(2).

(2) Let $M$ be a f.g. graded right $B$-module. Since $B_{R}$ is f.g., $M$ is a f.g. $R$ module. By (E1.5.1), GKdim $M$ is only dependent on the Hilbert series of $M$, not the module structure. Therefore GKdim $M_{B}=\operatorname{GKdim} M_{R}$.

\section{A Morita type Theorem}

The main result of this section is Theorem 2.4 We consider the following hypotheses to be used in different situations.

Hypothesis 2.1. (1) $A$ and $B$ are noetherian algebras.

(2) Let $e$ be an idempotent in $B$ and $A=e B e$.

(3) $\partial$ is a dimension function defined on right $A$-modules and $B$-modules and $\partial(B)=: d \geq 2$.

(4) $B$ is a $\partial-C M(2)$ algebra.

(5) $e B$ and Be are f.g. A-modules.

(6) $\partial$ is exact both on right $A$-modules and on right $B$-modules.

(7) For every f.g. right $B$-module $N, \partial\left((N e)_{A}\right)\left(=\partial\left(N \otimes_{B} B e\right)\right) \leq \partial\left(N_{B}\right)$.

Note that Hypothesis 2.1(7) is always satisfied if $\partial=$ GKdim (cf. KL, Proposition 5.6]).

Under Hypothesis 2.1(2), $B e$ is a right $A$-module and there is a natural algebra morphism

$$
\varphi: B \rightarrow \operatorname{End}_{A}(B e), \quad \varphi(b)\left(b^{\prime} e\right)=b b^{\prime} e
$$

induced by the left multiplication. In this section we will investigate when this morphism $\varphi$ is an isomorphism. A special case of this situation is the Auslander theorem [Yo, Lemma 10.8].

We need use some weaker versions of $\mathrm{CM}$ condition. Let $\partial$ be a dimension function and $\alpha$ be an integer.

(a) We say a noetherian algebra $B$ is $\partial-C M^{l}(\alpha)$ if for any f.g. right $B$-module $M$,

$$
\partial(M) \leq d-\alpha \Longrightarrow j(M) \geq \alpha
$$

(b) We say a noetherian algebra $B$ is $\partial-C M^{r}(\alpha)$ if for any f.g. right $B$-module $M$,

$$
\partial(M) \leq d-\alpha \Longleftarrow j(M) \geq \alpha .
$$

Then $B$ is $\partial-\mathrm{CM}(\alpha)$ if and only if it is both $\partial-\mathrm{CM}^{l}(\alpha)$ and $\partial-\mathrm{CM}^{r}(\alpha)$. 
Lemma 2.2. Let $(A, B)$ satisfy Hypothesis 2.1 $(1,2,3)$ and $\alpha$ be an integer such that $0<\alpha \leq d$. Suppose that $B$ is $\partial-C M^{l}(\alpha)$. Let $N$ be a f.g. right $A$-module. If $\partial\left(\operatorname{Tor}_{i}^{A}(N, e B)\right) \leq d-\alpha$ for all $i \leq \alpha-1$, then $\operatorname{Ext}_{A}^{i}(N, B e)=0$ for all $i \leq \alpha-1$.

Proof. We have canonical isomorphisms induced by the adjointness

$$
\operatorname{Hom}_{B}\left(N \otimes_{A} e B, B\right) \cong \operatorname{Hom}_{A}\left(N, \operatorname{Hom}_{B}(e B, B)\right) \cong \operatorname{Hom}_{A}(N, B e) .
$$

From these isomorphisms, we obtain a spectral sequence [Ro, Theorem 11.54]

$$
E_{2}^{p q}=\operatorname{Ext}_{B}^{p}\left(\operatorname{Tor}_{q}^{A}(N, e B), B\right) \Longrightarrow \operatorname{Ext}_{A}^{p+q}(N, B e) .
$$

By hypothesis, $B$ is $\partial-\mathrm{CM}^{l}(\alpha)$, we have $\operatorname{Ext}_{B}^{p}\left(\operatorname{Tor}_{q}^{A}(N, e B), B\right)=0$ for all $q \leq \alpha-1$ and $p \leq \alpha-1$ since $\partial\left(\operatorname{Tor}_{i}^{A}(N, e B)\right) \leq d-\alpha$ for all $i \leq \alpha-1$. By (E2.2.1), we obtain $\operatorname{Ext}_{A}^{i}(N, B e)=0$ for all $i \leq \alpha-1$.

In the next lemma it is not necessary to assume $d \geq 2$.

Lemma 2.3. Let $(A, B)$ satisfy Hypothesis 2.1(1-3),(5-7) and $\alpha$ be a positive integer. Suppose that

$$
\partial \text { satisfies } \gamma_{d-\alpha, 1}(e B) \text {. }
$$

If $\partial(B /(B e B)) \leq d-\alpha$, then the functor $-\otimes_{\mathcal{B}} \mathcal{B} e: \operatorname{qmod}_{d-\alpha} B \longrightarrow \operatorname{qmod}_{d-\alpha} A$ is an equivalence.

Proof. By hypothesis (E2.3.1), $\partial$ satisfies $\gamma_{d-\alpha, 1}(e B)$. By Lemma 1.3, we have the induced functor $-\otimes_{\mathcal{A}} e \mathcal{B}: \operatorname{qmod}_{d-\alpha} A \longrightarrow \operatorname{qmod}_{d-\alpha} B$. Since $B e$ is projective as a left $B$-module, the hypothesis of Lemma 1.3 follows from Hypothesis 2.1(7) with $M=B e$ and $A$ and $B$ are switched. By Lemma 1.3, we have the induced functor $-\otimes_{\mathcal{B}} \mathcal{B} e: \operatorname{qmod}_{d-\alpha} B \longrightarrow \operatorname{qmod}_{d-\alpha} A$. For any $N \in \bmod A$, we have natural isomorphisms in $\operatorname{qmod}_{d-\alpha} A$ :

$$
\mathcal{N} \otimes_{\mathcal{A}} e \mathcal{B} \otimes_{\mathcal{B}} \mathcal{B} e \cong \pi\left(N \otimes_{A} e B \otimes_{B} B e\right) \cong \pi(N)=\mathcal{N} .
$$

Taking $M \in \bmod B$, we have natural isomorphisms in $\operatorname{qmod}_{d-\alpha} B$ :

$$
\mathcal{M} \otimes_{\mathcal{B}} \mathcal{B} e \otimes_{\mathcal{A}} e \mathcal{B} \cong \pi\left(M \otimes_{B} B e \otimes_{A} e B\right) .
$$

We claim that $\pi\left(M \otimes_{B} B e \otimes_{A} e B\right)=\pi(M)$. Let $f: B e \otimes_{A} e B \rightarrow B$ be induced by the multiplication in $B$ and factor $f$ as $f=h g$ with $h$ a monomorphism and $g$ an epimorphism. Then we have two short exact sequences as follows,

$$
\begin{gathered}
0 \rightarrow K \rightarrow B e \otimes_{A} e B \stackrel{g}{\rightarrow} B e B \rightarrow 0, \\
0 \rightarrow B e B \stackrel{h}{\rightarrow} B \rightarrow B / B e B \rightarrow 0 .
\end{gathered}
$$

Applying the functor $-\otimes_{B} B e$ to (E2.3.2), we obtain the exact sequence

$$
0 \rightarrow \mathrm{Ke} \rightarrow \mathrm{Be} \otimes_{\mathrm{A}} \mathrm{eBe} \rightarrow \mathrm{Be} \rightarrow 0,
$$

which results that $K e=0$. Similarly, $e K=0$. Since $B e$ is a f.g. right $A$-module, $B e \otimes_{A} e B$ is a f.g. right $B$-module. Hence $K$ is a f.g. right $B / B e B$-module. Since $\partial(B / B e B) \leq d-\alpha$ and $\partial$ is exact on right $B$-modules, we have $\partial(K) \leq d-\alpha$. Consider the exact sequence

$$
M \otimes_{B} K \rightarrow M \otimes_{B} B e \otimes_{A} e B \rightarrow M \otimes_{B} B e B \rightarrow 0 .
$$


Since $M \otimes_{B} K$ is a f.g. $B / B e B$-module, $\partial\left(M \otimes_{B} K\right) \leq d-\alpha$. Now we have $\pi\left(M \otimes_{B} B e \otimes_{A} e B\right) \cong \pi\left(M \otimes_{B} B e B\right)$ in $\operatorname{qmod}_{d-\alpha} B$. From the exact sequence E2.3.3), we obtain an exact sequence

$$
\operatorname{Tor}_{1}^{B}(M, B / B e B) \rightarrow M \otimes_{B} B e B \rightarrow M \rightarrow M \otimes_{B} B / B e B \rightarrow 0 .
$$

Note that both $\operatorname{Tor}_{1}^{B}(M, B / B e B)$ and $M \otimes_{B} B / B e B$ are f.g. right $B / B e B$-modules. Since the $\partial$-dimension of $B / B e B$ is not larger than $d-\alpha$, neither are the $\partial$ dimensions of $M \otimes_{B} B / B e B$ and $\operatorname{Tor}_{1}^{B}(M, B / B e B)$. Hence we have natural isomorphisms in $\operatorname{qmod}_{d-\alpha} B: \pi\left(M \otimes_{B} B e B\right) \cong \pi(M)$. Summarizing, we obtain

$$
\mathcal{M} \otimes_{\mathcal{B}} \mathcal{B} e \otimes_{\mathcal{A}} e \mathcal{B} \cong \pi\left(M \otimes_{B} B e \otimes_{A} e B\right) \cong \pi(M)=\mathcal{M}
$$

as desired.

A special case of Lemma 2.3 is the following well-known statement: if $e$ is an idempotent in $B$ such that $B e B=B$, then $B$ is Morita equivalent to $A:=e B e$. Most of Hypothesis 2.1 is not necessary in this special case. Therefore Lemma 2.3 can be viewed as a generalization of Morita theorem. Specializing the last statement to a semisimple $H$-action on an algebra $A$, if $e:=1 \# \int$ in $B:=A \# H$ is such that $B e B=B$, then $A^{H}$ is Morita equivalent to $A \# H$, see [Mon, Corollary 4.5.4].

The main result of this section is the following Morita type theorem.

Theorem 2.4. Let $(A, B)$ satisfy Hypothesis 2.1(1-7). Suppose

$$
\text { d satisfies } \gamma_{d-2,1}(e B) \text {. }
$$

Then the following statements are equivalent.

(i) The functor $-\otimes_{\mathcal{B}} \mathcal{B} e: \operatorname{qmod}_{d-2} B \longrightarrow \operatorname{qmod}_{d-2} A$ is an equivalence.

(ii) The natural map $\varphi$ of E2.1.1 is an isomorphism of algebras.

(iii) $\partial(B /(B e B)) \leq d-2$.

(iv) $j(B / B e B) \geq 2$.

Proof. (i) $\Longrightarrow$ (ii). Let $F=-\otimes_{\mathcal{B}} \mathcal{B} e$. Since $F$ is an equivalence, we obtain isomorphisms of algebras:

$$
\operatorname{End}_{\mathrm{qmod}_{d-2} B} \mathcal{B} \cong \operatorname{End}_{\mathrm{qmod}_{d-2} A} A(\mathcal{B})=\operatorname{End}_{\mathrm{qmod}_{d-2} A} \pi(B e) .
$$

By assumption, $B$ is $\partial-\mathrm{CM}^{l}(2)$. Hence, for $M \in \bmod _{d-2} B$,

$$
\operatorname{Hom}_{B}(M, B)=0=\operatorname{Ext}_{B}^{1}(M, B) \text {. }
$$

As a consequence, ${ }_{B} B$ does not contain any nonzero submodule of $\partial$-dimension at most $d-2$. Therefore, by definition,

$$
\operatorname{End}_{\mathrm{qmod}_{d-2} B} \mathcal{B}=\underset{\longrightarrow}{\longrightarrow} \operatorname{lom}_{B}(K, B),
$$

where the limit runs over all the right submodule modules $K \subseteq B$ such that $\partial(B / K) \leq d-2$. For such a submodule $K$, we have

$$
\operatorname{Hom}_{B}(B / K, B)=0=\operatorname{Ext}_{B}^{1}(B / K, B) .
$$

Hence, a long exact sequence implies that $\operatorname{Hom}_{B}(K, B)=\operatorname{Hom}_{B}(B, B)=B$. Thus, we have $\operatorname{End}_{\mathrm{qmod}_{d-2} B} \mathcal{B} \cong B$.

Let $N \in \bmod _{d-2} A$. By Lemma 1.3(1) and (E2.4.1), the hypothesis of Lemma 2.2 concerning $\partial\left(\operatorname{Tor}_{i}^{A}(N, e B)\right)$ holds for $\alpha=2$. Hence, by Lemma 2.2.

$$
\operatorname{Hom}_{A}(N, B e)=0=\operatorname{Ext}_{A}^{1}(N, B e)
$$


for all $N \in \bmod _{d-2} A$. In particular, $B e$ does not have any nonzero right $A$ submodule of $\partial$-dimension $\leq d-2$. Using this and (E2.4.3), we have

$$
\operatorname{Hom}_{\mathrm{qmod}_{d-2} A}(\mathcal{B} e, \mathcal{B} e)=\underset{\longrightarrow}{\longrightarrow} \operatorname{Hom}_{A}(K, B e) \cong \operatorname{Hom}_{A}(B e, B e),
$$

where the limit runs over all the submodules $K \subseteq B e$ such that $\partial(B e / K) \leq d-2$. Combining with (E2.4.2), one sees that $B \cong \operatorname{Hom}_{A}(B e, B e)$, and the isomorphism is the natural morphism given in statement (ii).

(ii) $\Longrightarrow$ (iv). Let $f: B e \otimes_{A} e B \longrightarrow B$ be the $B$-bimodule morphism defined by $f\left(b e \otimes_{A} e b^{\prime}\right)=b e b^{\prime}$, which was used in the proof of Lemma 2.3. Consider the following composition of morphisms:

$$
\begin{aligned}
& B=\operatorname{Hom}_{B}(B, B) \stackrel{\operatorname{Hom}_{B}(f, B)}{\longrightarrow} \operatorname{Hom}_{B}\left(B e \otimes_{A} e B, B\right) \\
& \stackrel{\phi}{\longrightarrow} \operatorname{Hom}_{A}\left(B e, \operatorname{Hom}_{B}(e B, B)\right) \stackrel{\alpha}{\longrightarrow} \operatorname{Hom}_{A}(B e, B e),
\end{aligned}
$$

where $\phi$ is the natural isomorphism defined by the adjointness, and $\alpha$ is the isomorphism induced by the isomorphism $\operatorname{Hom}_{B}(e B, B) \cong B e$. One sees that the composition above is equal to the natural morphism

$$
\varphi: B \longrightarrow \operatorname{End}_{A}(B e), \quad \varphi(b)\left(b^{\prime} e\right)=b b^{\prime} e .
$$

By (ii), it is an isomorphism. Hence the morphism

$$
\operatorname{Hom}_{B}(f, B): \operatorname{Hom}_{B}(B, B) \longrightarrow \operatorname{Hom}_{B}\left(B e \otimes_{A} e B, B\right)
$$

is an isomorphism. Let us factor the morphism $f$ as follows:

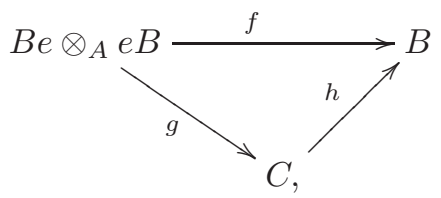

where $C=\operatorname{im} f=B e B, g$ is the epimorphism induced by $f$ and $h$ is the inclusion map. Applying the functor $\operatorname{Hom}_{B}(-, B)$ to the diagram above, we have $\operatorname{Hom}_{B}(f, B)=\operatorname{Hom}_{B}(g, B) \circ \operatorname{Hom}_{B}(h, B)$. Since $g$ is an epimorphism, it follows that $\operatorname{Hom}_{B}(g, B)$ is a monomorphism, and hence it has to be an isomorphism, which in turn implies that $\operatorname{Hom}_{B}(h, B)$ is an isomorphism. From the exact sequence

$$
0 \longrightarrow C \stackrel{h}{\longrightarrow} B \longrightarrow B / B e B \longrightarrow 0,
$$

we obtain the following exact sequence

$$
\begin{aligned}
& 0 \longrightarrow \operatorname{Hom}_{B}(B / B e B, B) \longrightarrow \operatorname{Hom}_{B}(B, B) \\
& \stackrel{\operatorname{Hom}_{B}(h, B)}{\longrightarrow} \operatorname{Hom}_{B}(C, B) \longrightarrow \operatorname{Ext}_{B}^{1}(B / B e B, B) \longrightarrow 0 .
\end{aligned}
$$

Since $\operatorname{Hom}_{B}(h, B)$ is an isomorphism, we obtain $\operatorname{Hom}_{B}(B / B e B, B)=0$ and $\operatorname{Ext}_{B}^{1}(B / B e B, B)=0$. Hence $j(B /(B e B)) \geq 2$.

(iv) $\Longrightarrow$ (iii) This follows from $\partial-\mathrm{CM}^{r}(2)$.

(iii) $\Longrightarrow$ (i). This is a special case of Lemma 2.3 when $\alpha=2$.

Remark 2.5. We have the following observations concerning the proof of Theorem 2.4 .

(1) In the proof of (i) $\Longrightarrow$ (ii), only $\partial-\mathrm{CM}^{l}(2)$ is used (not the entire $\partial-\mathrm{CM}(2)$ ). 
(2) In the proof of (iv) $\Longrightarrow$ (iii), only $\partial-\mathrm{CM}^{r}(2)$ is used (not the entire $\partial$ $\operatorname{CM}(2))$. In fact, we only used the fact that $j(B / B e B) \geq 2$ implies that $\partial(B / B e B) \leq d-2$.

(3) In the proof of (iii) $\Longrightarrow$ (i), $\partial-\mathrm{CM}(2)$ is not needed.

(4) (iii) $\Longrightarrow$ (iv) Follows from the definition of $\partial-\mathrm{CM}(2)$.

Now let $B=\bigoplus_{i \geq 0} B_{i}$ be a noetherian locally finite graded algebra. Let $e \in B_{0}$ be an idempotent. Then $A:=e B e$ is a noetherian locally finite graded algebra. Following Definition 1.4, $B$ is said to be graded $C M$ if GKdim $B=d<\infty$ and $j(M)+\mathrm{GK} \operatorname{dim} K=\mathrm{GK} \operatorname{dim} B$ for all f.g. graded right $B$-modules $M$.

Note that the GK-dimension is always exact on modules over noetherian locally finite graded algebras [Remark 1.5(2)]. By Lemma 1.6. GKdim satisfies $\gamma_{n, i}$. Note that, by (E1.5.1), GKdim satisfies Hypothesis 2.1(7) in the locally finite graded case. Therefore we obtain the following graded version of Theorem 2.4

Theorem 2.6. Let $B=\bigoplus_{i>0} B_{i}$ be a noetherian locally finite graded algebra. Suppose $(A, B)$ satisfies Hypothesis 2.1(1-5) in the graded setting with $e \in B_{0}$ and $\partial=$ GKdim. Then the following statements are equivalent.

(i) The functor $-\otimes_{\mathcal{B}} \mathcal{B} e: \operatorname{qgr}_{d-2} B \longrightarrow \operatorname{qgr}_{d-2} A$ is an equivalence.

(ii) The natural map $\varphi: B \longrightarrow \operatorname{End}_{A}(B e)$ defined by $\varphi(b)\left(b^{\prime} e\right)=b b^{\prime} e$ is an isomorphism of graded algebras.

(iii) $\operatorname{GKdim} B /(B e B) \leq d-2$.

(iv) $j(B / B e B) \geq 2$.

\section{Hopf AlgEBra ACTIONS ON GRADED ALGEBRAS}

In this section we consider locally finite graded algebras and let $\partial$ be a dimension function on locally finite graded modules. Starting from Proposition 3.3 , we further assume that $\mathbb{k}$ is a field.

Let $(H, \Delta, \varepsilon, S)$ be a Hopf algebra that is free of finite rank over $\mathbb{k}$. Let $R$ be a noetherian locally finite graded algebra. Assume that $R$ is a graded left $H$-module algebra, i.e., $H$ acts on $R$ homogeneously. Then the smash product $R \# H$ is a noetherian locally finite graded algebra.

Suppose that $H$ has a (left and right) integral $\int$ such that $\varepsilon\left(\int\right)=1$. If $\mathbb{k}$ is a field, this is equivalent to the fact that $H$ is semisimple. Let $e=1 \# \int \in R \# H$. One sees that $e$ is an idempotent of $R \# H$. Write the fixed subring of the $H$-action on $R$ as

$$
R^{H}=\{r \in R \mid h \cdot r=\varepsilon(h) r, \forall h \in H\} .
$$

Then $R^{H}$ is a graded subalgebra of $R$. The following is well-known Mon.

Lemma 3.1. With the notation as above. The following hold.

(1) $R^{H}$ is noetherian.

(2) $R$ is f.g. as a left graded $R^{H}$-module and as a right graded $R^{H}$-module.

(3) The map $R^{H} \longrightarrow e(R \# H)$ e, sending $r \mapsto e(r \# 1) e$, is an isomorphism of graded algebras.

(4) The map $R \longrightarrow(R \# H)$ e, sending $r \mapsto(r \# 1) e$, is an isomorphism of graded $\left(R, R^{H}\right)$-modules.

(5) The map $R \longrightarrow e(R \# H)$, sending $r \mapsto e(r \# 1)$, is an isomorphism of graded $\left(R^{H}, R\right)$-modules. 
Proof. (1) This follows by the proof of [Mon, Corollary 4.3.5].

(2) This follows by the proof of [Mon, Theorem 4.4.2].

$(3,4,5)$ By direct computation.

Hypothesis 3.2. $\quad$ (1) $R$ is a noetherian, locally finite, graded algebra.

(2) $H$ is a Hopf algebra acting on $R$ homogeneously, and $H$ is free of finite rank over $\mathbb{k}$ with integral $\int$ such that $\epsilon\left(\int\right)=1$.

(3) Let $B$ be the algebra $R \# H$ with $e:=1 \# \int \in B$. Identifying $R^{H}$ with eBe by Lemma 3.1(3), $R$ with Be by Lemma 3.17(5).

(4) Let $\partial$ be an exact dimension function on f.g. right graded B-modules, $R$ modules, and $R^{H}$-modules. This is automatic if $\partial=$ GKdim.

(5) $\partial$ is $B / R$-hereditary. This is automatic if $\partial=$ GKdim by Lemma 1.10(2).

(6) $\partial(R)=d \geq 2$ and $R$ is graded $\partial-C M(2)$

Here is our first result in this section. For the rest of this section we assume that $\mathbb{k}$ is a field.

Proposition 3.3. Retain Hypothesis 3.2(1-5). If $R$ is $\partial-C M$ (respectively, $\partial$ $\left.C M(s), \partial-C M^{l}(s), \partial-C M^{r}(s)\right)$, then so is $B:=R \# H$.

Proof. Since $B$ is isomorphic to $R^{\oplus \operatorname{dim} H}$ as a right $R$-module, we have

$$
\partial\left(B_{B}\right)=\partial\left(B_{R}\right)=\partial\left(R_{R}\right)=: d .
$$

Let $M$ be a f.g. graded right $B$-module with $\partial$-dimension $i$. Since $\partial$ is $B / R$ hereditary, $M$, viewed as a right $R$-module, has $\partial$-dimension $i$, or $\partial\left(M_{B}\right)=\partial\left(M_{R}\right)$. It remains to show that $j\left(M_{B}\right)=j\left(M_{R}\right)$.

The cohomology $\operatorname{Ext}_{B}^{j}(M, B)$ can be computed by $\operatorname{Ext}_{R}^{j}(M, B)$. Since $H$ is semisimple, by [Ne, Corollary 7.6], we have

$$
\underline{\operatorname{Ext}}_{B}^{j}(M, B) \cong \underline{\operatorname{Ext}}_{R}^{j}(M, B)^{H} \text {. }
$$

Equation (E3.3.1) follows also from a more general result [St, Theorem 3.3] together with [We, Lemma 9.1.9].

We recall the right $H$-module structure on $\underline{\operatorname{Ext}}_{R}^{j}(M, B)$ as follows. Let

$$
\cdots \longrightarrow P^{-n} \longrightarrow \cdots \longrightarrow P^{0} \longrightarrow M_{B} \longrightarrow 0
$$

be a f.g. projective resolution of $M_{B}$. Let $N$ be a right $B$-module. For $j \geq 0$, $\underline{\operatorname{Hom}}_{k}\left(P^{-j}, N\right)$ has a right $H$-module structure defined by

$$
(f \cdot h)(p)=f\left(p\left(S h_{(1)}\right)\right) h_{(2)}
$$

for all $p \in P^{-j}, f \in \underline{\operatorname{Hom}}_{\mathrm{k}}\left(P^{-j}, N\right)$ and $h \in H$, where $S$ is the antipode of $H$ and we use Sweedler's notation $\Delta(h)=h_{(1)} \otimes h_{(2)}$. One may check that $\underline{\operatorname{Hom}}_{R}\left(P^{-j}, N\right)$ is an $H$-submodule of $\underline{\operatorname{Hom}}_{k}\left(P^{-j}, N\right)$. The right $H$-action is compatible with the differential of the complex $\underline{\operatorname{Hom}}_{R}\left(P^{\bullet}, N\right)$, from which we obtain the right $H$-action on $\underline{\operatorname{Ext}}_{R}^{j}(M, N)$.

We may form another smash product $H \# R$ as follows [RRZ, Lemma 2.1]: as a vector space $H \# R=H \otimes R$, the product is defined by

$$
(h \# r)\left(g \# r^{\prime}\right)=h g_{(2)} \#\left(S^{-1} g_{(1)} \cdot r\right) r^{\prime} .
$$

The algebras $R \# H$ and $H \# R$ are isomorphic through the isomorphism:

$$
\varphi: R \# H \longrightarrow H \# R \text {, }
$$


defined by

$$
r \# h \mapsto h_{(2)} \# S^{-1} h_{(1)} \cdot r .
$$

For all $j \geq 0$, we have the following morphism

$$
\Psi: \underline{\operatorname{Hom}}_{R}\left(P^{-j}, R \# H\right) \longrightarrow \underline{\operatorname{Hom}}_{R}\left(P^{-j}, H \# R\right),
$$

defined by

$$
\Psi(f)=\varphi \circ f .
$$

We also may define a morphism

$$
\Phi: \underline{\operatorname{Hom}}_{R}\left(P^{-j}, R\right) \otimes H \longrightarrow \underline{\operatorname{Hom}}_{\mathbb{k}}\left(P^{-j}, H \# R\right),
$$

defined by

$$
\Phi(f \otimes h)(p)=h \# f(p) .
$$

A straightforward check shows that $\Phi$ takes value in $\underline{\operatorname{Hom}}_{R}\left(P^{-j}, H \# R\right)$. Hence, we indeed obtain a morphism $\Phi: \underline{\operatorname{Hom}}_{R}\left(P^{-j}, R\right) \otimes H \longrightarrow \underline{\operatorname{Hom}}_{R}\left(P^{-j}, H \# R\right)$. One may check that both $\Psi$ and $\Phi$ are isomorphisms of vector spaces and are compatible with the differentials. Through the isomorphism $\varphi$, we view $H \# R$ as a right $R \# H$-module. Note that $R$ can be viewed as a right $R \# H$-module via the action

$$
r \cdot\left(r^{\prime} \# h\right)=S^{-1} h\left(r r^{\prime}\right)
$$

for $r, r^{\prime} \in R$ and $h \in H$. Hence both $\underline{\operatorname{Hom}}_{R}\left(P^{-j}, H \# R\right)$ and $\underline{\operatorname{Hom}}_{R}\left(P^{-j}, R\right)$ are right $H$-modules. Now $\underline{\operatorname{Hom}}_{R}\left(P^{-j}, R\right) \otimes H$ is also a right $H$-module by the usual $H$-action defined by

$$
(f \otimes g) \cdot h=f \cdot h_{(1)} \otimes g h_{(2)}
$$

for $f \in \underline{\operatorname{Hom}}_{R}\left(P^{-j}, R\right)$ and $g, h \in H$. Since $\varphi$ is an isomorphism of algebras, $\Psi$ is an isomorphism of right $H$-modules. We next check that $\Phi$ is a right $H$ module morphism, and hence it is indeed an isomorphism of right $H$-modules. For $f \in \underline{\operatorname{Hom}}_{R}\left(P^{-j}, R\right), h, g \in H$ and $p \in P^{-j}$, we have

$$
\begin{aligned}
{[\Phi(f \otimes h) \cdot g](p) } & =\left[\Phi(f \otimes h)\left(p S g_{(1)}\right)\right] g_{(2)} \\
& =\left[h \# f\left(p S g_{(1)}\right)\right] g_{(2)} \\
& =h g_{(3)} \# S^{-1} g_{(2)} f\left(p\left(S g_{(1)}\right)\right) \\
\Phi((f \otimes h) g)(p) & =\Phi\left(f \cdot g_{(1)} \otimes h g_{(2)}\right)(p) \\
& =h g_{(2)} \#\left(f \cdot g_{(1)}\right)(p) \\
& =h g_{(3)} \# f\left(p S g_{(1)}\right) \cdot g_{(2)} \\
& =h g_{(3)} \# S^{-1} g_{(2)} f\left(p S g_{(1)}\right) .
\end{aligned}
$$

Hence $\Phi$ is a right $H$-module morphism. Therefore the composition $\Phi^{-1} \Psi$ induces an isomorphism of complexes of right $H$-modules

$$
\underline{\operatorname{Hom}}_{R}\left(P^{\bullet}, R \# H\right) \longrightarrow \underline{\operatorname{Hom}}_{R}\left(P^{\bullet}, R\right) \otimes H .
$$

Applying $(-)^{H}$, we obtain an isomorphism of complexes

$$
\underline{\operatorname{Hom}}_{R}\left(P^{\bullet}, R \# H\right)^{H} \cong\left(\underline{\operatorname{Hom}}_{R}\left(P^{\bullet}, R\right) \otimes H\right)^{H} .
$$

Since $(-)^{H}$ is an exact functor, taking cohomology $H^{j}(-)$ and taking the invariants $(-)^{H}$ commutes. Therefore we have

$$
\underline{\operatorname{Ext}}_{R}^{j}(M, R \# H)^{H} \cong\left(\underline{\operatorname{Ext}}_{R}^{j}(M, R) \otimes H\right)^{H} \text {. }
$$

Combining with the isomorphism (E3.3.1), we finally obtain

$$
\underline{\operatorname{Ext}}_{B}^{j}(M, B) \cong\left(\underline{\operatorname{Ext}}_{R}^{j}(M, R) \otimes H\right)^{H} \text {. }
$$


By (E3.3.2), it is clear that $j\left(M_{B}\right) \geq j\left(M_{R}\right)$. Assume that $x_{1}, \ldots, x_{n}$ is a $\mathbb{k}$ basis of $H$. Then we may write the coproduct of $\int$ as $\Delta\left(\int\right)=\sum_{s=1}^{n} y_{s} \otimes x_{s}$. Since

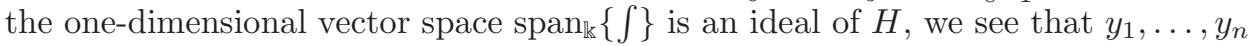
generate $H$ by $\left[\mathrm{Sw}\right.$, Proposition 2.6]. If $\underline{\operatorname{Ext}}_{R}^{j}(M, R) \neq 0$, there is a nonzero element $\alpha \in \underline{\operatorname{Ext}}_{R}^{j}(M, R)$ such that $\alpha y_{s} \neq 0$ for some $s$. Now $\sum_{s=1}^{n} \alpha y_{s} \otimes x_{s} \neq 0$, which is in $\left(\underline{\operatorname{Ext}}_{R}^{j}(M, R) \otimes H\right)^{H}$. Therefore $\underline{\operatorname{Ext}}_{B}^{j}(M, B) \neq 0$. This means that $j\left(M_{B}\right) \leq$ $j\left(M_{R}\right)$. Therefore $j\left(M_{B}\right)=j\left(M_{R}\right)$.

Combining Lemmas 2.2, 1.6 and 3.1 and Proposition 3.3. we have the following consequences.

Proposition 3.4. Retain Hypothesis $3.2(1,2,3)$ with $\partial=$ GKdim and assume that $\mathbb{k}$ is a field and that $R$ is $C M(\alpha)$ for a positive integer $\alpha$. Then $\underline{\operatorname{Ext}}_{R^{H}}^{i}(N, R)=0$ for all $N \in \operatorname{gr}_{d-\alpha} R^{H}$ and all $i \leq \alpha-1$.

Proof. Let $(A, B)=\left(R^{H}, R \# H\right)$. Then Hypothesis 2.1 $(1,2,3)$ can be verified easily. By Proposition 3.3. $B$ is $\operatorname{CM}(\alpha)$. The assertion follows from Lemmas [1.6 and 2.2 .

Assisted by Proposition 3.3. Theorem 2.6 now reads in the following form. Recall that the pertinency of $H$-action on $R$ [Definition 0.1] is defined to be

$$
\mathrm{p}(R, H)=\operatorname{GKdim} R-\operatorname{GKdim}(R \# H) /(e)
$$

where $e=1 \# \int$.

Theorem 3.5. Retain Hypothesis $3.2(1-3)$ with $\partial=$ GKdim. Assume that $R$ is $C M(2)$ with $\operatorname{GKdim} R=d \geq 2$. Set $(A, B)=\left(R^{H}, R \# H\right)$. Then the following are equivalent.

(i) The functor $-\otimes_{\mathcal{B}} \mathcal{R}: \operatorname{qgr}_{d-2} B \longrightarrow \operatorname{qgr}_{d-2} A$ is an equivalence.

(ii) The natural map $\varphi: B \longrightarrow \operatorname{End}_{A}(R)$ is an isomorphism of algebras.

(iii) $\mathrm{p}(R, H) \geq 2$.

(iv) $H$-action is homologically small in the sense of Definition 0.2(2).

Proof. This is an immediate consequence of Theorem 2.6 after matching up with notations and verifying the hypotheses by Proposition 3.3. Note that (iii) and (iv) are equivalent by $\mathrm{CM}(2)$.

Remark 3.6. (1) Theorem 0.3 is a special case of Theorem 3.5

(2) A version of Theorem 3.5 holds when $R$ is ungraded (or $R$ is not locally finite) as long as

(i) the GK-dimension on right $R^{H}$-modules are exact,

(ii) the GK-dimension is $R \# H / R^{H}$-hereditary,

(iii) $\gamma_{n, 1}(e B)$ holds for GKdim.

(3) If $R=\mathbb{C}\left[x_{1}, \ldots, x_{d}\right]$ is the polynomial algebra, and $G$ a finite small subgroup of $\mathrm{GL}_{d}(\mathbb{C})$, the Auslander theorem says the natural map $R \# \mathbb{k} G \longrightarrow \operatorname{End}_{R^{G}}(R)$ as in Theorem 3.5 is an isomorphism of graded algebras [Au1, Yo, IT]. By Theorem 3.5. $\mathrm{p}(R, G) \geq 2$. See comments in BHZ, Section 7].

(4) If $R / R^{H}$ is an $H^{*}$-dense Galois extension in the sense of [HVZ2, then $\mathrm{p}(R, H)=d$. In this case, [HVZ2, Theorems 2.4 and 3.8] implies that the statement (ii) of Theorem 3.5 holds. Hence the equivalence of (ii) and (iii) may be viewed as a generalization of [HVZ2, Theorem 3.8(ii)]. In particular, if $\mathrm{p}(R, H)=\mathrm{GKdim} R=$ 
2, then the equivalence of (i) and (ii) in Theorem 3.5 is indeed a part of HVZ2, Theorem 3.8]. On the other hand, [HVZ2, Theorem 3.8] doesn't assume that the algebra $R$ is CM; instead, there is an additional assumption on the depth of $R$ as a right $A$-module.

(5) If $G$ is a finite group of graded automorphisms of $R$, and $R$ is AS-regular and CM, then Theorem 3.5 implies [MU, Theorem 3.7] since the ampleness of the group action implies that $\mathrm{p}(R, H)=\operatorname{GKdim} R$. However, the isomorphism in MU, Theorem 3.7] holds for more general AS-regular algebras.

Next we prove Theorem 0.6

Theorem 3.7. Retain Hypothesis $3.2(1,2,3)$. Let $\alpha$ be an integer no more than $\mathrm{p}(R, H)$, and let $d=\operatorname{GKdim} R$. Then there is an equivalence of categories

$$
\operatorname{qgr}_{d-\alpha} R^{H} \cong \operatorname{qgr}_{d-\alpha} R \# H .
$$

Proof. By Lemma 1.6, hypothesis (E2.3.1) holds for $\partial=$ GKdim on graded modules. Hypotheses 2.1 (1-3)(5-7) can be checked one by one. Therefore the assertion follows by Lemma 2.3 .

Corollary 3.8. Retain Hypothesis $3.2(1,2,3)$. Let $R$ be an $A S$ regular algebra. If $\mathrm{p}(R, H)=\operatorname{GKdim} R$, then $R^{H}$ has graded isolated singularities.

Proof. Assume $d=\operatorname{GKdim} R$. By Theorem 3.7 $\operatorname{qgr}_{d-\alpha} R^{H} \cong \operatorname{qgr}_{d-\alpha} R \# H$ where the latter has finite global dimension. Taking $\alpha=d$, the assertion follows by the definition, see [Ue, Definition 2.2].

For the rest of this section we make some observations the condition to ensure that $\mathrm{p}(R, H) \geq 1$. We recall a definition below.

Definition 3.9. A left $H$-module $M$ is called inner faithful if $I M \neq 0$ for every nonzero Hopf ideal $I$ of $H$. We say that an $H$-action on $A$ is inner faithful, if $A$ is an inner faithful left $H$-module.

Lemma 3.10. Retain Hypothesis $3.2(1-5)$ with $\partial=$ GKdim. Assume that $\mathbb{k}$ is a field. Suppose that $R$ is a domain and $(A, B)=\left(R^{H}, R \# H\right)$.

(1) $\mathrm{SkV}$, Theorem $0.5(\mathrm{i})] B$ is semiprime.

(2) $\overline{\mathrm{BCF}}$, Corollary 3.4] $B$ is prime if and only if the canonical algebra homomorphism $\phi: B \rightarrow \operatorname{End}_{R^{H}}(R)$ is injective.

(3) If the map $\phi$ in part (2) is injective, then the $H$-action on $R$ is inner faithful. As a consequence, if $B$ is prime, then the $H$-action is inner faithful.

(4) $B$ is prime if and only if $\mathrm{p}(R, H) \geq 1$.

Proof. (1) This is a special case of [SkV, Theorem 0.5(i)].

(2) In order to apply $\mathrm{BCF}$, Corollary 3.4$]$ we need to verify the hypothesis in BCF, Corollary 3.4], namely, the condition in BCF, Lemma 3.3(2)]. Since $R$ is a domain, so is $A:=R^{H}$. Hence $A$ is prime. Let $I$ be a nonzero one-sided ideal of $R$ which is stable under the $H$-action. Since $R$ is a domain, $\operatorname{GKdim} R / I<\operatorname{GKdim} R$. If $A \cap I=0$, then

$$
\operatorname{GKdim} R / I=\operatorname{GKdim} A=\operatorname{GKdim} R,
$$

a contradiction. Hence $A \cap I \neq 0$. So, Condition [BCF, Lemma 3.3(2)] is satisfied. The assertion now follows from $\mathrm{BCF}$, Corollary 3.4]. 
(3) If the $H$-action on $R$ is not inner faithful, there is a Hopf ideal $0 \neq J \subseteq H$ such that $J R=0$. Thus $\phi(1 \# j)=0$ for all $j \in J$. The assertion follows. The consequence follows from part (2).

(4) Let $d=\operatorname{GKdim} R$. First we suppose that $\mathrm{p}(R, H) \geq 1$. By part (1), $B$ is semiprime. Since $B$ is a free module over $R$ and $R$ is a domain, $B$ is $d$-pure for $R$ modules, and hence for $B$-modules with respect to the GK-dimension in the sense of ASZ2, Definition 0.3]. By Lemma 1.6. Hypothesis E2.3.1 holds for any $\alpha$, and hence for $\alpha=1$. By Lemma $2.3 \operatorname{qmod}_{d-1} B \cong \operatorname{qmod}_{d-1} A$. Since $A$ is an Ore domain, $\operatorname{qmod}_{d-1} A$ is equivalent to a module category over the quotient division ring $Q(A)$ of $A$. Therefore, $\operatorname{qmod}_{d-1} B$ is equivalent to a module category over a division ring. Since $B$ is GKdim-pure, $\operatorname{qmod}_{d-1} B \cong \bmod Q(B)$ where $Q(B)$ is the semisimple artinian quotient ring of $B$. This implies that $Q(B)$ is simple and $B$ is prime.

Conversely, since $B$ is prime and $I=B e B \subseteq B$ is a nonzero ideal, GKdim $B / I \leq$ $d-1$. Hence $\mathrm{p}(R, H) \geq 1$.

Lemma 3.10 were suggested by Ellen Kirkman. The authors thank her for sharing her ideas.

\section{BGG CORRESPONDENCE}

From now on let $\mathbb{k}$ be a field. In this section, we study the quotient category $\operatorname{qgr}_{d-\alpha} R^{H}$ as appeared in the last section. If $R$ is noetherian, connected graded, AS regular and if the homological determinant of the $H$-action on $R$ is trivial, then $R^{H}$ is AS Gorenstein KKZ1, Theorem 0.1]. Under the setting of Theorem 3.5 (assuming $\mathrm{p}(R, H) \geq 2$ ), the quotient category $\operatorname{qgr}_{d-2} R^{H}$ is equivalent to the quotient category $\operatorname{qgr}_{d-2} R \# H$. Since $R \# H$ has finite global dimension, while $R^{H}$ has infinite global dimension when $H$ is non-trivial [CKWZ1, Theorem 0.6], it is relatively easier to understand homological properties of the category $\operatorname{qgr}_{d-2} R \# H$ than to do the category $\operatorname{qgr}_{d-2} R^{H}$ directly.

We are now working over Koszul AS regular algebras. Let $B=\bigoplus_{n>0} B_{n}$ be a noetherian locally finite graded algebra such that $B_{0}$ is a semisimple algebra. Recall that a right graded $B$-module is called a Koszul module [Pr, BGS] if $M$ has a linear graded projective resolution:

$$
\cdots \longrightarrow P^{-n} \longrightarrow \cdots \longrightarrow P^{-1} \longrightarrow P^{0} \longrightarrow M \longrightarrow 0,
$$

where $P^{-n}$ are graded projective modules generated in degree $n$ for all $n \geq 0$. If the right graded $B$-module $B_{0}:=B / B_{\geq 1}$ is a Koszul module, then $B$ is called a Koszul algebra.

Let $B$ be a graded algebra and let

$$
E=E(B):=\oplus_{n \geq 0} \underline{\operatorname{Ext}}_{B}^{n}\left(B_{0}, B_{0}\right)
$$

be the Yoneda algebra of $B$. By BGS, Proposition 2.9.1 and Theorem 2.10.1], if $B$ is a Koszul algebra, then the Yoneda algebra $E$ is also Koszul.

Let $B$ be a locally finite graded algebra. Let $\left\{S_{1}, \cdots, S_{w}\right\}$ be the complete set of non-isomorphic graded right $B$-module concentrated in degree 0 , and let $Q_{i}$ be the projective cover of $S_{i}$, for $i=1, \cdots, w$. Define

$$
B^{\prime}=\underline{\operatorname{Hom}}_{B}\left(\bigoplus_{i=1}^{w} Q_{i}, \bigoplus_{i=1}^{w} Q_{i}\right) .
$$


In applications we also assume that the degree zero piece $B_{0}$ of $B$ is semisimple. The following lemma is well-known.

Lemma 4.1. Retain the notations as above and assume that $B_{0}$ is semisimple.

(1) $B^{\prime}$ is graded Morita equivalent to B. Namely, there is an equivalence of graded module categories

$$
\operatorname{Gr} B^{\prime} \cong \mathrm{Gr} B,
$$

which induces an equivalence of triangulated categories

$$
D\left(\operatorname{Gr} B^{\prime}\right) \cong D(\operatorname{Gr} B) .
$$

(2) $E(B)$ is graded Morita equivalent to $E\left(B^{\prime}\right)$.

(3) $B$ is noetherian (respectively, AS Gorenstein, AS regular, Koszul) if and only if so is $B^{\prime}$.

(4) If $\mathbb{k}$ is algebraically closed, then $B_{0}^{\prime}$ is a finite direct sum of $\mathbb{k}$.

For the rest of this section, we assume that $\mathbb{k}$ is algebraic closed. Then $B_{0}^{\prime}$ is a finite direct sum of $\mathbb{k}$ [Lemma 4.1(4)]. In this case $B^{\prime}$ is called the basic algebra of $B$. Assume that $R$ is as in Theorem 3.5 and that $R$ is a connected graded Koszul AS regular algebra. In view of [HVZ1, Theorem 3.2], $R \# H$ is a Koszul algebra with finite dimensional Koszul dual. Hence $R \# H$ has finite global dimension. By E3.3.2 and its dual version, it follows that $R \# H$ is an AS-regular algebra. Since the graded Morita equivalence of algebras preserves almost all algebraic invariants [Lemma 4.1, we will study the basic algebra of $R \# H$ instead. In general, one can replace $B$ by its basic algebra $B^{\prime}$ if necessary.

From now on, $B=B_{0} \oplus B_{1} \oplus \cdots$ is a noetherian locally finite graded algebra such that $B_{0}$ is a finite direct sum of $\mathbb{k}$. The following lemma is needed.

Lemma 4.2. [Ma, Theorem 5.1] Let $B$ be a Koszul algebra. Then $B$ is AS regular if and only if $E:=\bigoplus_{n \geq 0} \underline{\operatorname{Ext}}_{B}^{n}\left(B_{0}, B_{0}\right)$ is a graded Frobenius algebra.

Let $B$ be a Koszul algebra. We may construct a differential bigraded algebra $\widetilde{B}$ by setting $\widetilde{B}_{j}^{0}=B_{j}$ and $\widetilde{B}_{j}^{i}=0$ for all $i \neq 0$ with zero differential. We may construct a differential bigraded algebra $\widetilde{E}$ associated to $E$ (E4.0.1) by setting $\widetilde{E}_{-i}^{i}=E_{i}$ and $\widetilde{E}_{j}^{i}=0$ for all $i \neq-j$ with zero differential. A differential bigraded $\widetilde{B}$-module $M$ is a bigraded $\widetilde{B}$-module $M=\oplus_{i, j \in \mathbb{Z}} M_{j}^{i}$ together with a differential $d$ such that $d\left(M_{j}^{i}\right) \subseteq M_{j}^{i+1}$ for all $i, j \in \mathbb{Z}$. Note that the derived category of differential bigraded right $\widetilde{B}$-modules (respectively, $\widetilde{E}$-modules) is equivalent to the derived category of right graded $B$-modules (respectively, $E$-modules). Then the general Koszul duality of bigraded differential graded algebras induces following duality between the derived category of $B$ and of $E$ [HW, Remark 4.1], which is a slightly different from the equivalence obtained in BGS]. Note that Smith-Van den Bergh stated a similar result in $\mathrm{SmV}$.

Proposition 4.3. SmV, Section 2.4] Let B be a Koszul AS regular algebra. We have a duality of triangulated categories:

$$
K: D^{b}(\operatorname{gr} B) \longrightarrow D^{b}(\operatorname{gr} E)
$$

where $K(M)=G\left(\mathrm{RHom}_{B}\left(M, B_{0}\right)\right)$ for $M \in D^{b}(\operatorname{gr} B)$, and $G$ is the regrading functor defined by $G(X)_{s}^{n}=X_{-s}^{n+s}$ for any complex of graded modules $X$. 
Note that the functor $K$ commutes with the shift functors in the following way

$$
K\left(X^{\cdot}[1]\right)=K\left(X^{\cdot}\right)[-1] \text { and } K\left(X^{\cdot}(1)\right)=K\left(X^{\cdot}\right)[-1](1),
$$

where as usual [1] is the shift of the complexes and (1) is the shift of the gradings.

For $M \in \operatorname{gr} E$, let $\Omega(M)$ be the first syzygy of the minimal graded projective resolution of $M$. Since $E$ is a graded Frobenius algebra, $\Omega$ is an auto-equivalence of the stable category $\operatorname{gr} E$. Moreover, $\operatorname{gr} E$ is a triangulated category with the shift functor $\Omega^{-1}$. We next show that the triangulated category gr $E$ admits some thick triangulated subcategories. Some terminologies are needed next. Let $M$ be a finite dimensional right graded $E$-module. Take a minimal graded projective resolution of $M$ as follows

$$
\cdots \longrightarrow P^{-n} \longrightarrow \cdots \longrightarrow P^{0} \longrightarrow M \longrightarrow 0 .
$$

Recall that the complexity of $M$, denoted by $c(M)$, is the least integer $\lambda \geq 0$ such that $\operatorname{dim} P^{-n}<a n^{\lambda-1}$ for almost all $n$, where $a>0$ is a fixed number. The definition of the complexity can be modified even for noninteger $\lambda$. Note that $c(M)=0$ if and only if $M$ is a finite dimensional graded projective $E$-module (when $E$ is Frobenius). The complexity of a module over a finite dimension algebra is an important invariant in studying the representations of the algebra [Ca, CDW, GLW.

Given a number $\lambda \geq 0$, let $\mathcal{C}_{\lambda}$ be the full subcategory of $\operatorname{gr} E$ consisting of objects $M$ with $c(M) \leq \lambda$. Similar to CDW, Proposition 1.3], we have the following lemma, whose proof is almost the same as that of CDW Proposition 1.3].

Lemma 4.4. The induced category $\mathcal{C}_{\lambda}$ is a thick triangulated subcategory of $\underline{\operatorname{gr}} E$.

Proof. Let $0 \rightarrow K \rightarrow M \rightarrow N \rightarrow 0$ be an exact sequence in gr $E$. Then one sees $c(M) \leq \max \{c(K), c(N)\}$. Hence $M \in \mathcal{C}_{\lambda}$ if $K$ and $N$ are in $\mathcal{C}_{\lambda}$. Moreover, it follows from [AR, Lemma 2.5] or [GLW, Lemma 2.7] that if $K$ and $M$ (resp. $M$ and $N$ ) are in $\mathcal{C}_{\lambda}$, then so is $N$ (resp. $K$ ). Note that the projective modules and injective module coincide in the category gr $E$ and any projective module has zero complexity. If $U \rightarrow V \rightarrow Q \rightarrow \Omega^{-1}(U)$ is a triangle in $\operatorname{gr} E$ with $U, V \in \mathcal{C}_{\lambda}$, then both $Q$ and $\Omega^{-1} U$ are in $\mathcal{C}_{\lambda}$, where $\Omega^{-1}(U)$ is the first syzygy of $U$ by taking minimal graded injective resolution of $U$. Hence $\mathcal{C}_{\lambda}$ is a triangulated subcategory of $\operatorname{gr} E$. Now if $N$ is a direct summand of $M$ in $\operatorname{gr} E$, then $N$ is a direct summand of $\bar{M} \oplus P$ in gr $E$ for some finite dimensional projective module $P$. Thus, we have $c(N) \leq c(M)$. Therefore $\mathcal{C}_{\lambda}$ is thick.

Let $D^{b}(\operatorname{proj} E)$ be the full subcategory of $D^{b}(\operatorname{gr} E)$ consisting of all bounded complexes of f.g. graded projective $E$-modules. Then $D^{b}(\operatorname{proj} E)$ is equivalent to the triangulated subcategory of $D^{b}(\operatorname{gr} E)$ generated by $E$. Since $E$ is a graded Frobenius algebra, there is a well known equivalence of triangulated categories [Be, Corollary 3.9(c)]

$$
\Phi: D^{b}(\operatorname{gr} E) / D^{b}(\operatorname{proj} E) \longrightarrow \underline{\operatorname{gr}} E,
$$

where the functor $\Phi$ is defined in the following way. Let $X^{\cdot}$ be an object in $D^{b}(\operatorname{gr} E)$. We write $\bar{X}$ for its image in $D^{b}(\operatorname{gr} E) / D^{b}(\operatorname{proj} E)$. Take a bounded above f.g. projective resolution $P^{\cdot}$ of $X^{*}$. Since $X^{\cdot}$ is bounded, we see that there is an integer $n$ such that the cohomology $H^{i} P^{\cdot}=0$ for all $i \leq-n$. Now, let $M$ be the $n$th syzygy of the complex $P$. Note that in the triangulated category $D^{b}(\operatorname{gr} E) / D^{b}(\operatorname{proj} E)$, 
the complex $P$ is isomorphic to its naive truncation $P \leq-n$, which is isomorphic to $M[n-1]$. Hence $\Phi\left(\overline{X^{*}}\right)=\Omega^{1-n}(M)$ Or, Sect. 1.3].

One can easily extend the notion of the complexity to bounded complexes of modules over a finite dimensional algebra. Let $\Lambda:=\Lambda_{0} \oplus \Lambda_{1} \oplus \cdots$ be a finite dimensional graded algebra such that $\Lambda_{0}$ is a semisimple algebra. Let $K^{-}(\operatorname{gr} \Lambda)$ be the homotopy category of bounded above complexes of f.g. right graded $\Lambda$ modules. A bounded above complex of graded projective modules $P^{\cdot} \in K^{-}(\operatorname{gr} \Lambda)$ is called minimal if im $d^{n}$ is superfluous, namely, im $d^{n} \subseteq P^{n} \Lambda_{\geq 1}$ for all $n$, where $\Lambda_{\geq 1}=\Lambda_{1} \oplus \Lambda_{2} \oplus \cdots$. Note that, for any bounded complex $X \cdot$ of f.g. right graded $\Lambda$-modules, there is a minimal complex of graded projective modules $P$ that is quasi-isomorphic to $X$. Such $P$ is called a minimal projective resolution of $X$. It is known that minimal projective resolution of a bounded complex of f.g. graded $\Lambda$-module is unique up to isomorphism. We define the complexity $c\left(X^{*}\right)$ of $X^{\cdot}$ to be the least integer $\lambda$ such that $\operatorname{dim} P^{-n} \leq a n^{\lambda-1}$ for almost all $n \geq 0$. If two bounded complexes $X$ and $Y^{\cdot}$ are quasi-isomorphic, then their minimal projective resolutions are isomorphic. Hence $c\left(X^{*}\right)=c\left(Y^{*}\right)$. Hence, it makes sense to define the complexity of an object in $D^{b}(\operatorname{gr} A)$. For $\lambda \geq 0$, we denote by $D_{\lambda}^{b}(\operatorname{gr} \Lambda)$ the full subcategory of $D^{b}(\operatorname{gr} \Lambda)$ consisting of objects $X^{\cdot}$ such that $c\left(X^{*}\right) \leq \lambda$. One sees that $D_{0}^{b}(\operatorname{gr} \Lambda)$ is equivalent to $D^{b}(\operatorname{proj} \Lambda)$.

Lemma 4.5. Retain the above notation. Then $D_{\lambda}^{b}(\operatorname{gr} \Lambda)$ is a thick triangulated subcategory of $D^{b}(\operatorname{gr} \Lambda)$ for all $\lambda \geq 0$.

Proof. Take $X^{\cdot}, Y^{\cdot} \in D_{\lambda}^{b}(\operatorname{gr} \Lambda)$. Let $f: X^{\cdot} \rightarrow Y^{\cdot}$ be a morphism in $D^{b}(\operatorname{gr} \Lambda)$, and $X^{\cdot} \rightarrow Y^{\cdot} \rightarrow Z^{\cdot} \rightarrow X^{\cdot}[1]$ be a triangle. Let $P^{\cdot}$ and $Q^{\cdot}$ be minimal projective resolutions of $X^{\cdot}$ and $Y$ respectively. The morphism $f$ induces a morphism $g$ : $P^{\cdot} \rightarrow Q$. Then cone $(g) \cong Z$ in $D^{b}(\operatorname{gr} \Lambda)$. Hence the minimal projective resolution of $Z$ is a direct summand of cone $(g)$. Therefore, $c(Z) \leq c($ cone $(g)) \leq \lambda$, and hence $D_{\lambda}^{b}(\operatorname{gr} \Lambda)$ is a triangulated subcategory of $D^{b}(\operatorname{gr} \Lambda)$. It is clear that $D_{\lambda}^{b}(\operatorname{gr} \Lambda)$ is closed under taking direct summands.

We can also extend the notion of the GK-dimension from modules to bounded complexes. Let $S$ be a noetherian locally finite graded algebra. Let $X$ be a bounded complex of right graded $S$-modules. Set $H^{\cdot}\left(X^{*}\right)=\bigoplus_{n \in \mathbb{Z}} H^{n}\left(X^{\cdot}\right)$, the total cohomology of $X^{\cdot}$. Define the GK-dimension of $X^{\cdot}$ as

$$
\operatorname{GKdim} X^{\cdot}:=\operatorname{GKdim} H^{\cdot}\left(X^{\cdot}\right) \text {. }
$$

By definition, if two complexes $X^{\cdot}$ and $Y^{\cdot}$ are quasi-isomorphic, then GKdim $X^{*}=$ GKdim $Y$. Hence it makes sense to say the GK-dimension of an object in the bounded derived category. For $\lambda \geq 0$, let $D_{\operatorname{gr}_{\lambda}}^{b}(\operatorname{gr} S)$ be the full subcategory of $D^{b}(\operatorname{gr} S)$ consisting of objects $X^{\cdot}$ such that $\operatorname{GKdim} X^{\cdot} \leq \lambda$. One sees that $X^{\cdot} \in$ $D_{\operatorname{gr}_{\lambda}}^{b}(\operatorname{gr} S)$ if and only if GKdim $H^{n}\left(X^{\cdot}\right) \leq \lambda$ for all $n \in \mathbb{Z}$. Hence we have the following.

Lemma 4.6. For every $\lambda \geq 0, D_{\mathrm{gr}_{\lambda}}^{b}(\mathrm{gr} S)$ is a thick triangulated subcategory of $D^{b}(\operatorname{gr} S)$.

We now go back to the $H$-action on a noetherian connected graded AS regular algebra $R$.

Setup 4.7. Throughout the rest of this section, we assume that $R$ is a noetherian connected graded AS regular algebra which is also a Koszul algebra. Let $B$ be the 
graded basic algebra of $R \# H$. Recall that $\mathbb{k}$ is assumed to be algebraically closed. Then $B_{0} \cong \mathbb{k}^{\oplus w}$ for some $w>0$ [Lemma 4.1(4)]. Let $E=\oplus_{n \geq 0} \underline{\operatorname{Ext}}_{B}^{n}\left(B_{0}, B_{0}\right)$ as given in (E4.0.1).

Lemma 4.8. Let $M$ be a right Koszul B-module. Then $\operatorname{GKdim}(M)=c(K(M))$.

Proof. Since $B$ is a Koszul algebra, $K(M)$ is a Koszul $E$-module, moreover, $K(M)$ has a minimal projective resolution [GM, Proposition 5.1]

$$
\cdots \longrightarrow P^{-n} \longrightarrow \cdots \longrightarrow P^{-1} \longrightarrow P^{0} \longrightarrow K(M) \longrightarrow 0
$$

such that $P^{-n} \cong M_{n}^{*} \otimes_{E_{0}} E$, where $M_{n}^{*}=\operatorname{Hom}\left(M_{n}, \mathbb{k}\right)$.

Assume $c(K(M))=\lambda$. Then $\operatorname{dim} M_{n} \leq \operatorname{dim} P^{-n} \leq a n^{\lambda-1}$ for some fixed number $a>0$ and for all $n \gg 0$. Hence $\operatorname{GKdim}(M) \leq \lambda$.

Conversely, assume $\operatorname{GKdim}(M)=\lambda$. By assumption $B$ is the basic algebra of $R \# H$. Hence there is a finitely generated graded progenerator $R \# H$-module $Q$, where $Q:=\bigoplus_{i=1}^{w} Q_{i}$ as given in $\left(\underline{E 4.0 .2}\right.$, such that $\underline{\operatorname{End}}_{R \# H}(Q) \cong B$. Write $F=-\otimes_{R \# H} Q$. Assume $N$ is a graded right $R \# H$-module such that $F(N) \cong M$. Then $\operatorname{GKdim}(N)=\operatorname{GKdim}(M)=\lambda$ by Remark 1.5(3). Note that $N$ is also a graded right $R$-module and by assumption $R$ is a noetherian connected graded AS regular algebra. By ATV, Proposition 2.21] and [StZ, Corollary 2.2], we have $\operatorname{dim} N_{n} \leq a n^{\lambda-1}$ for some fixed number $a>0$ and for all $n \gg 0$. Since $M=F(N)$, there is an $a_{1}>0$ such that $\operatorname{dim} M_{n} \leq a_{1} \operatorname{dim} N_{n} \leq a_{1} a n^{\lambda-1}$ for all $n \gg 0$. Assume $\operatorname{dim} E=b$. Then $\operatorname{dim} P^{-n} \leq b \operatorname{dim} M_{n} \leq b a_{1} a n^{\lambda-1}$. Hence $c(K(M)) \leq \lambda$.

Lemma 4.9. For every integer $\lambda \geq 0$, the Koszul duality given in Proposition 4.3 restricts to the following anti-equivalence of triangulated categories,

$$
D_{\operatorname{gr}_{\lambda}}^{b}(\operatorname{gr} B) \cong D_{\lambda}^{b}(\operatorname{gr} E) .
$$

Proof. Firstly, notice that if $M$ is a f.g. Koszul graded $B$-module, then, by Proposition 4.3. $H^{i}(K(M))=0$ for $i \neq 0$. In particular, $K\left(B_{0}\right) \cong E$. Hence we see that the thick triangulated subcategory of $D^{b}(\mathrm{gr} B)$ generated by $B_{0}$ is anti-equivalent to the thick triangulated subcategory of $D^{b}(\mathrm{gr} E)$ generated by $E$. The former is equivalent to $D_{\mathrm{gr}_{0}}^{b}(\mathrm{gr} B)$ and the latter is equivalent to $D^{b}(\operatorname{proj} E)$ which is in turn equivalent to $D_{0}^{b}(\operatorname{gr} E)$. So we have proven the assertion for $\lambda=0$.

Next we assume that $\lambda \geq 1$. By the Koszul duality, we only need to show the following:

(a) If $X^{\cdot} \in D_{\operatorname{gr}_{\lambda}}^{b}(\operatorname{gr} B)$, then $K\left(X^{\cdot}\right) \in D_{\lambda}^{b}(\operatorname{gr} E)$.

(b) If $Y^{\cdot} \in D_{\lambda}^{b}(\operatorname{gr} E)$, then $K^{-1}\left(Y^{\cdot}\right) \in D_{\operatorname{gr}_{\lambda}}^{b}(\operatorname{gr} B)$.

Since $D_{\operatorname{gr}_{\lambda}}^{b}(\operatorname{gr} B)$ and $D_{\lambda}^{b}(\operatorname{gr} E)$ are thick triangulated subcategories, it suffices to show that,

(c) for every f.g. right graded $B$-modules $M$ with $\operatorname{GKdim} \leq \lambda$, we have $K(M) \in$ $D_{\lambda}^{b}(\operatorname{gr} E)$, and

(d) for every f.g. right graded $E$-modules $N$ such that $c(N) \leq \lambda$, we have $K^{-1}(N) \in D_{\operatorname{gr}_{\lambda}}^{b}(\operatorname{gr} B)$.

Let $M$ be a f.g. right $B$-module with $\operatorname{GKdim} M \leq \lambda$. Since $B$ is a Koszul AS-regular algebra, it follows that for sufficiently large $k$ the graded $B$-module $M_{\geq k}(k)$, where $M_{\geq k}=M_{k} \oplus M_{k+1} \oplus \cdots$, has linear resolution (see [Jo1, Theorem 3.1], note that it is assumed that the algebra is connected graded in [Jo1, however, the same proof 
holds in our case), that is, $M_{\geq k}(k)$ is a Koszul module. Consider the exact sequence

$$
0 \rightarrow M_{\geq k} \rightarrow M \rightarrow M / M_{\geq k} \rightarrow 0
$$

We see that $\operatorname{GKdim} M_{\geq k}=\operatorname{GKdim} M \leq \lambda$ and

$$
K\left(M / M_{\geq k}\right) \rightarrow K(M) \rightarrow K\left(M_{\geq k}\right) \rightarrow K\left(M / M_{\geq k}\right)[1]
$$

is a triangle in $D^{b}(\operatorname{gr} E)$. Since $M / M_{\geq k}$ is finite dimensional, $K\left(M / M_{\geq k}\right) \in$ $D_{0}^{b}(\operatorname{gr} E)$. Since $M_{\geq k}(k)$ is a Koszul module, $c\left(K\left(M_{\geq k}\right)\right)=\operatorname{GKdim}\left(M_{\geq k}\right) \leq \lambda$ by Lemma 4.8. Hence $K\left(M_{\geq k}\right) \in D_{\lambda}^{b}(\operatorname{gr} E)$. Therefore $K(M) \in D_{\lambda}^{b}(\operatorname{gr} E)$. This verifies claim (c).

For $Y^{\cdot} \in D_{\lambda}^{b}(\operatorname{gr} E)$, let $P^{\cdot}$ be a minimal projective resolution of $Y^{\cdot}$. Then $\operatorname{dim} P^{-n} \leq a n^{\lambda-1}$ for $n \gg 0$. Since $B_{0}$ is a direct sum of finitely many copies of $\mathbb{k}$, so is $E_{0}$. Assume that $E_{0}=\mathbb{k} \oplus \cdots \oplus \mathbb{k}$ is the sum of $s$ copies of $\mathbb{k}$, and that $e_{1}, \ldots, e_{s}$ are the corresponding primitive idempotents. Then $E=\oplus_{i=1}^{s} E e_{i}$. Assume $r=\min \left\{\operatorname{dim} E e_{i} \mid i=1, \ldots, s\right\}$. By the minimality of $P^{\prime}$, we see that

$$
\operatorname{dim} P^{-n} \geq r \sum_{j \in \mathbb{Z}} \operatorname{dim} \operatorname{Hom}_{D^{b}(\operatorname{gr} E)}\left(Y^{\prime}, E_{0}[n](j)\right) .
$$

Hence

$$
\sum_{j \in \mathbb{Z}} \operatorname{dim} \operatorname{Hom}_{D^{b}(\operatorname{gr} E)}\left(Y^{\cdot}, E_{0}[n](j)\right) \leq \frac{a}{r} n^{\lambda-1} .
$$

Given $i$ and $j$, in the following computations, note that $K(B) \cong E_{0}$ in $D^{b}(\operatorname{gr} E)$,

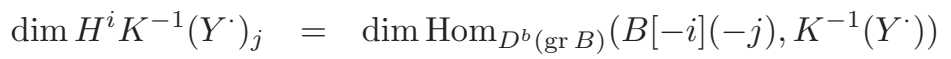

$$
\begin{aligned}
& =\operatorname{dim} \operatorname{Hom}_{D^{b}(\operatorname{gr} E)}\left(Y^{\cdot}, K(B[-i](-j))\right) \\
& =\operatorname{dim} \operatorname{Hom}_{D^{b}(\operatorname{gr} E)}\left(Y^{\cdot}, E_{0}[i+j](-j)\right) \\
& \leq \frac{a}{r}(i+j)^{\lambda-1} \text {. }
\end{aligned}
$$

Hence $\operatorname{GKdim} H^{i} K^{-1}\left(Y^{\cdot}\right) \leq \lambda$ for all $i$. Therefore, $K^{-1}\left(Y^{\cdot}\right) \in D_{\mathrm{gr}_{\lambda}}^{b}(\operatorname{gr} B)$. Thus we verify claim (b) (and (d)).

Lemma 4.10. There are equivalences of triangulated categories:

$$
D_{\lambda}^{b}(\operatorname{gr} E) / D^{b}(\operatorname{proj} E) \cong \mathcal{C}_{\lambda}
$$

for all $\lambda \geq 0$.

Proof. By Lemma 4.5, $D_{\lambda}^{b}(\mathrm{gr} E)$ is a thick triangulated subcategory of $D^{b}(\operatorname{gr} E)$ for each $\lambda \geq 0$. One sees that $D_{\lambda}^{b}(\operatorname{gr} E) / D^{b}(\operatorname{proj} E)$ is a full triangulated subcategory of $D^{b}(\operatorname{gr} E) / D^{b}(\operatorname{proj} E)$ for each $\lambda \geq 1$. Consider the equivalence

$$
\Phi: D^{b}(\operatorname{gr} E) / D^{b}(\operatorname{proj} E) \longrightarrow \underline{\operatorname{gr} E}
$$

as established by (E4.4.1). By the definition of $\Phi$, one easily sees that if $c\left(X^{*}\right) \leq \lambda$ then $c\left(\Phi\left(X^{\cdot}\right)\right) \leq \lambda$. Hence $\Phi$ sends objects in $D_{\lambda}^{b}(\operatorname{gr} E) / D^{b}(\operatorname{proj} E)$ to objects in $\mathcal{C}_{\lambda}$. If $N$ is a right graded $E$-module such that $c(N) \leq \lambda$, then we may view $N$ as an object in $D_{\lambda}^{b}(\operatorname{gr} E) / D^{b}(\operatorname{proj} E)$, and we have $\Phi(N) \cong N \in \mathcal{C}_{\lambda}$. Therefore the restriction of $\Phi$ to $D_{\lambda}^{b}(\operatorname{gr} E) / D^{b}(\operatorname{proj} E)$ is essential dense onto $\mathcal{C}_{\lambda}$, and hence $D_{\lambda}^{b}(\operatorname{gr} E) / D^{b}(\operatorname{proj} E)$ is equivalent to $\mathcal{C}_{\lambda}$.

We are ready to prove the main result of this section, which may be viewed as a generalization of the classical BGG correspondence BGG, Jo2, Mor1. 
Theorem 4.11. Let $B$ and $E$ be as in Setup 4.7. Then, for each $\lambda \geq 0$, there is an anti-equivalence of triangulated categories

$$
D^{b}\left(\operatorname{qgr}_{\lambda} B\right) \cong \underline{\operatorname{gr}} E / \mathcal{C}_{\lambda} .
$$

Proof. By the Koszul duality in Proposition 4.3, we have an anti-equivalence $K$ : $D^{b}(\operatorname{gr} B) \longrightarrow D^{b}(\operatorname{gr} E)$. Lemma 4.9 and its proof show that $K$ restricts to antiequivalences $D_{\mathrm{gr}_{\lambda}}^{b}(\operatorname{gr} B) \cong D_{\lambda}^{b}(\operatorname{gr} E)$ for each $\lambda \geq 0$. Hence we obtain antiequivalences

$$
D^{b}(\operatorname{gr} B) / D_{\operatorname{gr}_{\lambda}}^{b}(\operatorname{gr} B) \cong D^{b}(\operatorname{gr} E) / D_{\lambda}^{b}(\operatorname{gr} E)
$$

for each $\lambda \geq 0$. By the equivalence $\Phi: D^{b}(\operatorname{gr} E) / D^{b}(\operatorname{proj} E) \longrightarrow \underline{\operatorname{gr}} E$ and Lemma 4.10, we have

$$
D^{b}(\operatorname{gr} E) / D_{\lambda}^{b}(\operatorname{gr} E) \cong \underline{\operatorname{gr}} E / \mathcal{C}_{\lambda}
$$

On the other hand, by [Mi, Theorem 3.2] we have the equivalence of triangulated categories

$$
D^{b}(\operatorname{gr} B) / D_{\operatorname{gr}_{\lambda}}^{b}(\operatorname{gr} B) \cong D^{b}\left(\operatorname{qgr}_{\lambda} B\right)
$$

since $\operatorname{gr}_{\lambda} B$ is a Serre subcategory of gr $B$. Combine the (anti-)equivalences above, we finally obtain the anti-equivalences as required.

Remark 4.12. In the case when $\lambda=0$, we have $\mathcal{C}_{0}=0$ and $\operatorname{qgr}_{0} B=\operatorname{qgr} B$, the quotient category of gr $B$ modulo by the Serre subcategory of finite dimensional graded $B$-modules. Then the anti-equivalence in the theorem above has the form

$$
D^{b}(\operatorname{qgr} B) \cong \underline{\operatorname{gr} E}
$$

which is the classical BGG correspondence established in BGG, Jo2, Theorem 3.1] and [Mor1, Theorem 5.3].

Corollary 4.13. Assume Setup 4.7, Let $R$ be $C M(2)$ of $\mathrm{GKdim} d \geq 2$. If $R \# H \cong$ $\operatorname{End}_{R^{H}}(R)$, then there is a canonical anti-equivalence of triangulated categories

$$
D^{b}\left(\operatorname{qgr}_{d-2} R^{H}\right) \cong \underline{\operatorname{gr} E} / \mathcal{C}_{d-2}
$$

where $E=\bigoplus_{n \geq 0} \operatorname{Ext}_{R \# H}^{n}(H, H)$.

Proof. Note that $R$ and $H$ are in the situation as in Theorem 3.5

Assume that $R \# H \cong \operatorname{End}_{R^{H}}(R)$, we will show the category equivalence. Take $B$ to be the basic algebra of $R \# H$. Then $R \# H$ and $B$ are graded Morita equivalence. Since $R$ is a Koszul algebra, $E$ and $E^{\prime}:=\bigoplus_{n>0} \operatorname{Ext}_{B}^{n}\left(B_{0}, B_{0}\right)$ are also graded Morita equivalent [Lemma 4.1(2)]. Since the graded Morita equivalence preserves the GK-dimension, we have that $\operatorname{gr}_{\lambda}(R \# H)$ and $\operatorname{gr}_{\lambda} B$ are equivalent. As a consequence, $D^{b}\left(\operatorname{qgr}_{\lambda} B\right)$ is equivalent to $D^{b}\left(\operatorname{qgr}_{\lambda} R \# H\right)$. Combining this with Theorems 3.5 and 4.11, we have

$$
D^{b}\left(\operatorname{qgr}_{d-2} R^{H}\right) \cong D^{b}\left(\operatorname{qgr}_{d-2} R \# H\right) \cong D^{b}\left(\operatorname{qgr}_{d-2} B\right) \cong \underline{\operatorname{gr}} E^{\prime} / \mathcal{C}_{d-2} \cong \underline{\operatorname{gr}} E / \mathcal{C}_{d-2} .
$$

Proof of Theorem 0.7. . (1) is Theorem 4.11 (2) follows from Theorems 4.11 and 3.7. 


\section{Proof of Theorem 0.5}

In this section let $\mathbb{k}$ be a field and char $\mathbb{k} \nmid 2 n$. We consider some finite group actions on the $(-1)$-skew polynomial algebra $\mathbb{k}_{-1}\left[x_{1}, \ldots, x_{n}\right]$ and prove Theorem 0.5. We need a few lemmas.

Let $R$ be a graded domain. Let $G$ be a finite group and $H$ its dual group algebra $(\mathbb{k} G)^{\circ}$ acting on $R$ homogeneously. Assume that the $H$-action on $R$ is inner faithful. If $R$ is generated in degree 1 , then this is equivalent to that $H$-action on $R_{1}$ is inner faithful. In this setting, $R$ is also $\mathbb{Z} \times G$-graded. Forgetting the $\mathbb{Z}$-grading, $R$ is $G$-graded and $R$ is not $G^{\prime}$-graded for any proper subgroups $G^{\prime} \subsetneq G$. Write $R=\oplus_{g \in G} R_{g}$. Part (4) of the next lemma is due to [CKWZ2]. Let $I$ be the ideal (e) in $B:=R \# H$ where $e=1 \# \int$ and $\int$ is the integral of $H$ such that $\epsilon\left(\int\right)=1$.

Lemma 5.1. Let $R$ be a connected graded domain. Let $H:=(\mathbb{k} G)^{\circ}$ act on $R$ inner faithfully and write $R=\oplus_{g \in G} R_{g}$.

(1) For every $g \in G, R_{g} \neq 0$.

(2) $R$ is a free $H$-module.

(3) If $f \in \bigcap_{g \in G} R R_{g}$, then $f \# 1 \in I$.

(4) CKWZ2, Lemma 4.8(2)] Suppose $f_{1}, \cdots, f_{m} \in R$ are $G$-homogeneous elements in $R$ such that $\left\{h_{w} \mid w=1, \cdots, m\right\}=G$ where

$$
h_{w}:=\operatorname{deg}_{G}\left(f_{w}\right) \cdots \operatorname{deg}_{G}\left(f_{m}\right) .
$$

Then $f_{1} \cdots f_{m} \# 1 \in I$.

(5) Suppose $d:=\operatorname{GKdim} R<\infty$. Then $\mathrm{p}(R, H) \geq 1$. As a consequence, $R \# H$ is prime and the algebra map

$$
R \# H \rightarrow \operatorname{End}_{R^{H}}(R)
$$

is injective.

(6) $\mathrm{p}(R, H) \geq d-\operatorname{GKdim} R /\left(\bigcap_{g \in G} R R_{g}\right) \geq d-\max \left\{\operatorname{GKdim} R / R R_{g} \mid g \in G\right\}$.

(7) If there is an integer $s$ such that $R R_{g} \supseteq R_{\geq s}$ for all $g \in G$, then $\mathrm{p}(R, H)=$ $d$.

Proof. (1) Let $X=\left\{g \in G \mid R_{g} \neq 0\right\}$. The Hopf ideal associated with $X$ is $J=\left\{f \in(\mathbb{k} G)^{\circ} \mid f(X)=0\right\}$. Since $R$ is a domain, $X$ is a subsemigroup of $G$. Since $X$ is finite, it is a group. By the inner faithfulness $J=0$, and hence $X=G$.

(2) We may assume $R \neq \mathbb{k}$. Since $R$ is a connected graded domain, $R$ is infinite dimensional. As a consequence, $R^{H}$ is infinite dimensional. So each $R_{g}$ is infinite dimensional. So $R$ is a free $H$-module of infinite rank.

(3) Let $\left\{g_{1}, \cdots, g_{m}\right\}$ be the set $G$ where $g_{1}$ is the unit of $G$. The dual basis $\left\{p_{g_{1}}, \cdots, p_{g_{m}}\right\}$ is the complete set of orthogonal idempotents of $H$ that forms a $\mathbb{k}$-linear basis of $H$. The integral of $H$ is $p_{g_{1}}$ and $\sum_{i=1}^{m} p_{g_{i}}$ is the algebra unit of $H$. The coproduct of $H$ is determined by

$$
\Delta\left(p_{g}\right)=\sum_{h \in G} p_{h} \otimes p_{h^{-1} g}
$$

for all $g \in G$. Let $f \in R$ be a $G$-homogeneous element of degree $g \in G$. Then

$$
e(f \# 1)=\left(1 \# p_{g_{1}}\right)(f \# 1)=\sum_{h} p_{h}(f) \# p_{h^{-1}}=p_{g}(f) \# p_{g^{-1}}=f \# p_{g^{-1}} .
$$

This implies that

$$
f \# p_{g^{-1}} \in I
$$


for all $f \in R_{g}$. Since $I$ is a left $R$-module, we have

$$
R R_{g} \# p_{g^{-1}} \subseteq I
$$

for all $g \in G$. If $f \in \bigcap_{g \in G} R R_{g}$, then $f \# p_{g^{-1}} \in I$ for all $g \in G$. Then $f \# 1=$ $\sum_{g \in G} f \# p_{g^{-1}} \in I$.

(4) Let $f=f_{1} \cdots f_{m}$. Then $f_{w} \cdots f_{m} \in R_{h_{w}}$ and $f \in R R_{h_{w}}$ for all $w$. By hypothesis $\left\{h_{w}\right\}_{w=1}^{m}$ equals $G$. Then $f \in \bigcap_{g \in G} R R_{g}$. The assertion follows by part (3).

(5) Assume $|G|=m$ and write $G=\left\{g_{1}, \ldots, g_{m-1}, g_{m}=e\right\}$. By Part (1), we may choose $0 \neq f_{m} \in R_{e}$ and $0 \neq f_{i} \in R_{g_{i} g_{i+1}^{-1}}$ for $i=1, \ldots, m-1$. Then $f_{1}, \ldots, f_{m}$ satisfy the condition in part (4). Since $R$ is a domain, $f:=f_{1} \cdots f_{m} \neq 0$. By part (4), $f \# 1 \in I$. Then $B /(I)$ has GK-dimension no more than the GKdim $R-1$, or equivalently, $\mathrm{p}(R, H) \geq 1$. The assertion follows by Lemma 3.10 $(2,4)$.

(6) This follows from Lemma 5.2 below and the fact that the image of $R \rightarrow$ $R \# H /(I)$, denoted by $R^{\prime}$, is a quotient of $R /\left(\bigcap_{g \in G} R R_{g}\right)$, by part (3), and that there is an embedding $R /\left(\bigcap_{g \in G} R R_{g}\right) \rightarrow \bigoplus_{g \in G} R /\left(R R_{g}\right)$.

(7) This is an immediate consequence of (6).

Let $T$ be a subalgebra of $R$ such that $R_{T}$ and ${ }_{T} R$ are f.g..

Lemma 5.2. Let $R^{\prime}$ be the image of the map $R \rightarrow B \rightarrow B / I$ and $T^{\prime}$ be the image of $T$ in $R^{\prime}$. Then $\operatorname{GKdim} T^{\prime}=\operatorname{GKdim} R^{\prime}=\operatorname{GKdim} B / I$.

Proof. This follows from the fact that $B / I$ is f.g. over $R^{\prime}$.

Aiming for a proof of Theorem 0.5 , we now let $R$ be the $(-1)$-skew polynomial ring $\mathbb{k}_{-1}\left[x_{1}, \cdots, x_{n}\right]$. Let $H$ be the group algebra $\mathbb{k} W$ where $W$ is generated by the permutation $\sigma: x_{i} \rightarrow x_{i+1}, x_{n} \rightarrow x_{1}$ for all $1 \leq i \leq n-1$. Theorem 0.5 asserts that $R \# \mathbb{k} W \cong \operatorname{End}_{R^{W}}(R)$.

Let $\xi$ be a fixed primitive $n$th root of unity. We might assume that $\xi \in \mathbb{k}$ since $\mathrm{p}(R, H)$ is preserved by field extensions. Let $y_{j}=\sum_{i=1}^{n} \xi^{i j} x_{i}$, for $j=0,1, \ldots, n-1$. Then $\left\{y_{0}, \cdots, y_{n-1}\right\}$ is a basis of $R_{1}$. It is clear that $\sigma\left(y_{j}\right)=\xi^{-j} y_{j}$. Let $T$ be the central subalgebra of $R$ generated by $\left\{x_{i}^{2}\right\}_{i=1}^{n}$ and let $Y_{j}=\sum_{i=1}^{n} \xi^{i j} x_{i}^{2}$ for $j=0,1, \ldots, n-1$. Then $\left\{Y_{i}\right\}_{i=0}^{n-1}$ is a basis of the vector space $\oplus_{i=1}^{n} \mathbb{k} x_{i}^{2}$. It is clear that $R$ is a f.g. module over the central subalgebra $T$.

The group algebra $\mathbb{k} W$ can be viewed as a $(\mathbb{k} G)^{\circ}$ where $G$ is the character group of $W$. Note that $G \cong W$ as abstract groups. Let $g$ be a generator of $G$ such that $\operatorname{deg}_{G}\left(y_{1}\right)=g$. Then $\operatorname{deg}_{G}\left(y_{i}\right)=g^{i}$ for all $0 \leq i \leq n-1$.

Let $U_{n}=\left\{(0,1),(1,2), \cdots,\left(\left\lfloor\frac{n}{2}\right\rfloor-1,\left\lfloor\frac{n}{2}\right\rfloor\right)\right\}$. For any integer $i$, let $\bar{i}$ be the integer between 0 and $n-1$ such that $\bar{i}=i \bmod n$. The following lemma is easy to check.

Lemma 5.3. Retain the above notation for $R$. Let $i, j, k$ be integers (between 0 and $n-1$ modulo $n)$.

(1) $y_{i} y_{j}+y_{j} y_{i}=2 Y_{i+j}=2 Y_{\overline{i+j}}$.

(2) $y_{k} y_{j} y_{i}=-y_{k} y_{i} y_{j}+\left(y_{i} y_{j}+y_{j} y_{i}\right) y_{k}$.

(3) $R^{\prime} /\left(Y_{i} \mid i=0, \cdots, n-1\right)$ is finite dimensional.

(4) If $n$ is even and $i \geq \frac{n}{2}$, then $y_{i}^{2}=y_{j}^{2}$ where $j=i-\frac{n}{2}$. 
(5) Assume $n$ is even. If $i<j$ and $(i, j) \notin U_{n}$

$$
y_{j} y_{i}= \begin{cases}-y_{i} y_{j}+2 y_{s}^{2} & i+j=\text { even and }<n, s=\frac{i+j}{2}, \\ -y_{i} y_{j}+2 y_{s}^{2} & i+j=\text { even and } \geq n, s=\frac{i+j-n}{2}, \\ -y_{i} y_{j}+\left(y_{s} y_{s+1}+y_{s+1} y_{s}\right) & i+j=\text { odd and }<n, s=\frac{i+j-1}{2}, \\ -y_{i} y_{j}+\left(y_{s} y_{s+1}+y_{s+1} y_{s}\right) & i+j=\text { odd and }>n, s=\frac{i+j-1-n}{2} .\end{cases}
$$

(6) Suppose $n=2^{d}$. For each $s,\left(y_{s} y_{s-1}\right)^{n}=0$ in $R^{\prime}$, and hence in $R^{\prime \prime}:=$ $R^{\prime} /\left(y_{i}^{2} \mid i=0, \cdots, \frac{n}{2}\right)$.

(7) Suppose $n=2^{d}$. Then $R^{\prime \prime}$ is finite dimensional.

(8) Suppose $n=2^{d}$. Then $y_{s}^{2(d+1)}=0$ in $R^{\prime}$ for all $s$.

Proof. (1) By a direct computation.

(2) This follows from part (1) and the fact $Y_{\overline{i+j}}$ is central in $R$.

(3) This follows from Lemma 5.2 and the fact $T /\left(Y_{i} \mid i=0, \cdots, n-1\right)=k$.

(4) Follows from the computations in the proof of part (1).

From now on we assume that $n$ is even.

(5) It follows from part (1).

(6) Since $\operatorname{deg}_{G}\left(y_{s} y_{s-1}\right)$ is an odd power of $g$, it is a generator of $G$, and hence the assertion follows from Lemma 5.1(4).

(7) We use Diamond lemma. Fix an order $y_{0}<y_{1}<\cdots<y_{n-2}<y_{n-1}$ in $R^{\prime \prime}$, and extend it to the lexicographical order on all monomials.

By part (5), for any $j>i, y_{j} y_{i}$ can be written as a linear combination of lower terms. By part (4), $y_{i}^{2}$ when $i \geq \frac{n}{2}$ can be expressed as a lower term. By part (2), if $k>j>i, y_{k} y_{j} y_{i}$ can be written as a linear combination of lower terms.

Let $X$ be a reduced monomial basis of $R^{\prime \prime}$, which means that if $X^{\prime}$ is another monomial basis of $R^{\prime \prime}$, then, for any monomial $f \in X$, there is a monomial $f^{\prime} \in X^{\prime}$ with the same length as $f$ such that either $f=f^{\prime}$ or $f$ is lower than $f^{\prime}$. Let $0 \neq f=y_{i_{1}} y_{i_{2}} \cdots y_{i_{h}} \in X$. Then $i_{\alpha} \neq i_{\alpha+1}$ since $y_{i}^{2}=0$ in $R^{\prime \prime}$ for all $i$. Since there are only $n$ possible $i_{\alpha}$, the maximal length of increasing $i_{\alpha}$ is at most $n$. If $i_{\alpha}>i_{\alpha+1}$, then, only the third or forth case of part (5) may occur, and hence $i_{\alpha} \leq \frac{n}{2}$ and $i_{\alpha+1}=i_{\alpha}-1$, namely, $\left(i_{\alpha+1}, i_{\alpha}\right) \in U_{n}$.

We next prove that there are at most $\frac{1}{2} n^{2}$ pairs $\left(i_{\alpha+1}, i_{\alpha}\right) \in U_{n}$ appeared in $f$. Suppose we have two pairs $\left(i_{\alpha+1}, i_{\alpha}\right)$ and $\left(i_{\beta+1}, i_{\beta}\right)$ in $U_{n}$ with $\alpha<\beta$. By part (2), $\beta \neq \alpha+1$. If $\beta>\alpha+2$, then $i_{s}$ are strictly increasing between $i_{\alpha+1}$ and $i_{\beta}$ (up to taking $\beta$ minimal). So $i_{\beta} \geq i_{\alpha}+1$. If $\beta=\alpha+2$, then $i_{\beta} \geq i_{\alpha}$. Now let $Y_{i}=y_{i} y_{i-1}$ for all $i \leq \frac{n}{2}$. By part (6), $Y_{i}^{n}=\left(y_{i} y_{i-1}\right)^{n}=0$ in $R^{\prime \prime}$. The above argument shows that $f$ is of the form

$$
y_{i_{1}}^{d_{1}} Y_{i_{2}}^{e_{1}} y_{i_{3}}^{d_{2}} Y_{i_{4}}^{e_{2}} \cdots y_{i_{2 w-1}}^{d_{w}} Y_{i_{2 w}}^{e_{w}}
$$

where

$$
i_{1}<i_{2} \leq i_{3}<i_{4} \leq i_{5} \ldots
$$

and where $0 \leq d_{s} \leq 1$ and $0 \leq e_{s} \leq n-1$. By definition, $1 \leq i_{2}<i_{4}<$ $\cdots \leq \frac{n}{2}$. Therefore there are at most $\frac{n}{2} \cdot n$ pairs of $\left(i_{\alpha+1}, i_{\alpha}\right) \in U_{n}$ appeared in $f$. Consequently, the degree of $f$ is uniformly bounded. This means that $X$ is finite and $R^{\prime \prime}$ has finite dimension.

(8) By part (4), we may assume $s<\frac{n}{2}$. If $s$ is odd (which is coprime to $n$ ), then, by Lemma 5.14 (4), $y_{s}^{n}=0$ in $R^{\prime}$. 
Now we consider general $s$. By Lemma $5.1(3)$, it suffices to show that $y_{s}^{2(d+1)} \in$ $R R_{g^{i}}$ for any $i=1, \cdots, n-1$. Note that $y_{s}^{2(d+1)} \in R=R R_{g^{0}}$ is clear. Fix $i$ and write $\equiv$ to be $=\bmod R R_{g^{i}}$. We start with $y_{i} \equiv 0$. Then

$$
2 y_{s}^{2}=y_{i} y_{2 s-i}+y_{2 s-i} y_{i} \equiv y_{i} y_{2 s-i} \text {. }
$$

We prove by induction that, for each $w$,

$$
\left(2 y_{s}\right)^{2 w} \equiv y_{j_{1}} y_{j_{2}} \cdots y_{j_{w}} y_{i_{w}} \cdots y_{i_{2}} y_{i_{1}}
$$

where

$$
i_{\alpha}=2^{\alpha} s-2^{\alpha-1} i, \text { and } j_{\alpha}=-2^{\alpha} s+2^{\alpha-1} i+2 s .
$$

When $w=1$, this is (E5.3.1). Suppose (E5.3.2) holds for $w-1 \geq 1$. Then

$$
\begin{aligned}
\left(2 y_{s}\right)^{2 w} & =\left(2 y_{s}^{2}\right)\left(2 y_{s}\right)^{2(w-1)} \equiv\left(2 y_{s}^{2}\right) y_{j_{1}} y_{j_{2}} \cdots y_{j_{w-1}} y_{i_{w-1}} \cdots y_{i_{2}} y_{i_{1}} \\
& \equiv y_{j_{1}} y_{j_{2}} \cdots y_{j_{w-1}}\left(2 y_{s}^{2}\right) y_{i_{w-1}} \cdots y_{i_{2}} y_{i_{1}} \\
& \equiv y_{j_{1}} y_{j_{2}} \cdots y_{j_{w-1}}\left(y_{i_{w}} y_{j_{w}}+y_{j_{w}} y_{i_{w}}\right) y_{i_{w-1} \cdots y_{i_{2}} y_{i_{1}}} \\
& \equiv y_{j_{1}} y_{j_{2}} \cdots y_{j_{w-1}}\left(y_{j_{w}} y_{i_{w}}\right) y_{i_{w-1}} \cdots y_{i_{2}} y_{i_{1}}
\end{aligned}
$$

since $y_{s}^{2}$ is central in $R$ and $y_{j_{w}} y_{i_{w-1}} \cdots y_{i_{2}} y_{i_{1}} \in R_{g^{i}}$. Therefore (E5.3.1) follows from the induction. Now take $w=d$, we have

$$
\operatorname{deg}_{G}\left(y_{i_{d}} \cdots y_{i_{1}}\right)=g^{\left(2^{d+1}-2\right) s-\left(2^{d}-1\right) i}=g^{-2 s+i}
$$

which implies that $\operatorname{deg}_{G}\left(y_{s}^{2} y_{i_{d}} \cdots y_{i_{1}}\right)=g^{\left(2^{d+1}-2\right) s-\left(2^{d}-1\right) i}=g^{i}$ and

$$
\begin{aligned}
\left(2 y_{s}\right)^{2(d+1)} & \equiv\left(2 y_{s}^{2}\right) y_{j_{1}} y_{j_{2}} \cdots y_{j_{d-1}} y_{j_{d}} y_{i_{d}} y_{i_{d-1}} \cdots y_{i_{2}} y_{i_{1}} \\
& \equiv 2 y_{j_{1}} y_{j_{2}} \cdots y_{j_{d-1}} y_{j_{d}}\left(y_{s}^{2} y_{i_{d}} y_{i_{d-1}} \cdots y_{i_{2}} y_{i_{1}}\right) \equiv 0 .
\end{aligned}
$$

Since char $\mathbb{k} \nmid 2 n, y_{s}^{2(d+1)} \in R R_{g^{i}}$ for all $i$ as desired.

Proposition 5.4. Let $R$ be the $(-1)$-skew polynomial ring $\mathbb{k}_{-1}\left[x_{1}, \cdots, x_{n}\right]$ for $n \geq$ 2. Let $H$ be the group algebra $\mathbb{k} W$ where $W$ is generated by the permutation $\sigma$ : $x_{i} \rightarrow x_{i+1}, x_{n} \rightarrow x_{1}$ for all $1 \leq i \leq n-1$. Suppose $n=2^{d}$ for some $d \geq 1$.

(1) $\mathrm{p}(R, H)=\mathrm{GK} \operatorname{dim} R=n \geq 2$.

(2) $\operatorname{qgr}_{0} R \# H \cong \operatorname{qgr}_{0} R^{H}$.

(3) $R^{H}$ has graded isolated singularities in the sense of [Ue, Definition 2.2].

Proof. (1) By Lemma 5.2, it suffices to show that GKdim $R^{\prime}=0$. Let $\mathfrak{p}$ be a minimal prime ideal of $R^{\prime}$ and let $S=R^{\prime} / \mathfrak{p}$. For any $s, y_{s}^{2}$ is normal in $R^{\prime}$. By Lemma $5.3(8),\left(y_{s}^{2}\right)^{(d+1)}=0$ in $R^{\prime}$. Since $S=R^{\prime} / \mathfrak{p}$ is prime, $y_{s}^{2}=0$ in $S$. By Lemma 5.3 (7), $S$ is finite dimensional (and hence $S \cong \mathbb{k}$ ). Note that every noetherian algebra has finitely many minimal prime ideals GW, Theorem 3.4]. As $R^{\prime}$ is semiprime, it can be embedded into the product $\bigoplus_{\mathfrak{p}} R^{\prime} / \mathfrak{p}$ where $\mathfrak{p}$ runs over all the minimal prime ideals of $R^{\prime}$. Therefore $R^{\prime}$ is finite dimensional and GKdim $R^{\prime}=0$ as desired.

(2) This is a consequence of Theorem 0.6

(3) This follows from the definition and part (2).

Remark 5.5. We have some comments concerning the above computation.

(1) It is a little bit surprising that if $n=2^{d}$, then $\mathrm{p}(R, H)=\operatorname{GKdim} R$. However conjecture 0.9 would give an explanation of this phenomena. 
(2) We conjecture that when $n \neq 2^{d}$, then $\mathrm{p}(R, H)<\operatorname{GKdim} R$ and $R^{H}$ does not have graded isolated singularities. It would be nice if there is a formula for $\operatorname{dim}_{\text {sing }}\left(R^{H}\right)$ in terms of $n$.

In practice, we assume that $H$ acts on $A$ inner faithfully. When $R$ is an AS regular domain, we conjecture that $\mathrm{p}(R, H) \geq 1$, see Lemma 3.10. By Theorem 3.5. when $\mathrm{p}(R, H) \geq 2$, then the Auslander theorem holds. By Lemma 2.3 when $\mathrm{p}(R, H)=\alpha$ (or more generally $\mathrm{p}(R, H) \geq \alpha$ ), then $\operatorname{qgr}_{d-\alpha} R \# H \cong \operatorname{qgr}_{d-\alpha} R^{H}$ where $d=\operatorname{GKdim} R$. Since $R \# H$ is regular, this equivalence gives some bounds on the dimension of singular locus of $R^{H}$, see (E0.6.1). Therefore $\mathrm{p}(R, H)$ relates several other properties and invariants of the $H$-action on $R$.

The definition of reflection number $\mathrm{r}(R, G)$ is given in Definition 0.8 , Recall that $G$ is called conventionally small if $\mathrm{r}(R, G) \geq 2$. If the Conjecture 0.9 holds, then Auslander theorem holds for classical small groups by Theorem 3.5 .

Remark 5.6. (1) By definition, $\mathrm{r}\left(R, G^{\prime}\right) \geq \mathrm{r}(R, G)$ if $G^{\prime}$ is a subgroup of $G$.

(2) Suggested by part (1), we conjecture that $\mathrm{p}\left(R, H^{\prime}\right) \geq \mathrm{p}(R, H)$ if $H^{\prime}$ is a Hopf subalgebra of $H$ (assuming $H^{\prime}$-action on $R$ is still inner faithful).

(3) It is not clear to us if there is any relationship between $\operatorname{dim}_{\text {sing }}\left(R^{H^{\prime}}\right)$ and $\operatorname{dim}_{\text {sing }}\left(R^{H}\right)$. Suggested by part (2), we would guess that $\operatorname{dim}_{\text {sing }}\left(R^{H^{\prime}}\right) \leq$ $\operatorname{dim}_{\text {sing }}\left(R^{H}\right)$, but we don't have any result to support the claim.

We can compute some bounds for $\mathrm{p}(R, G)$ and $\mathrm{r}(R, G)$ in the following situation.

Theorem 5.7. Let $R$ be the (-1)-skew polynomial ring $\mathbb{k}_{-1}\left[x_{1}, \cdots, x_{n}\right]$ for $n \geq 2$. Let $H$ be the group algebra $\mathbb{k} W$ where $W$ is generated by the permutation $\sigma: x_{i} \rightarrow$ $x_{i+1}, x_{n} \rightarrow x_{1}$ for all $1 \leq i \leq n-1$.

(1) If $n=2^{d}$, then $\mathrm{p}(R, W)=n$.

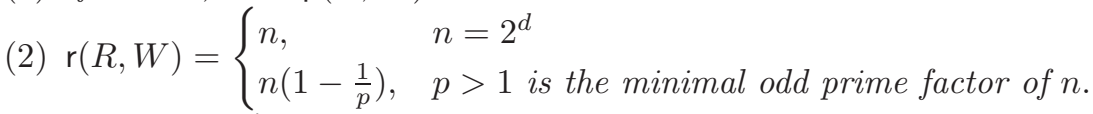

(3) $\mathrm{p}(R, W) \geq \begin{cases}\phi(n), & 4 \nmid n \\ \frac{\phi(n)}{2}, & 4 \mid n\end{cases}$ where $\phi(n):=n \prod_{\text {all primes } p \mid n}\left(1-\frac{1}{p}\right)$ is the Euler's totient function.

(4) If $n=p^{d}$ where $p$ is a prime number, then

$$
\mathrm{p}(R, W) \geq \mathrm{r}(R, W)= \begin{cases}n, & p=2, \\ n\left(1-\frac{1}{p}\right), & p>2 .\end{cases}
$$

As a consequence, Conjecture 0.9 holds in this case.

Proof. (1) This is Proposition 5.4

(2) Let $\sigma$ be the cycle permutation sending $x_{i} \rightarrow x_{i+1}$ for all $i<n$ and sending $x_{n}$ to $x_{1}$. Let $g$ be $\sigma^{i}$ for some $i<n$. Then $g$ is a product of $a$-many of disjoint cycles of length $b$ where $a b=n$. Let $o(g)$ be the order of the pole of $\operatorname{Tr}(g, t)$ at $t=1$. By KKZ2, Lemma 1.7], $o(g)$ is equal to the number of odd cycles. (The authors of [KKZ2 assume that char $\mathbb{k}=0$, however, [KKZ2, Lemma 1.7] holds when char $\mathbb{k} \neq 2$.) Hence $o(g)=a=n / b$ when $b$ is odd. If $n$ is of the form $2^{d}$, then $o(g)=0$ and $\mathrm{r}(R, G)=n$. If $n$ contains some odd factor, then the maximal possible $o(g)$ attains when $b$ is the smallest odd prime factor of $n$. Therefore $\mathrm{r}(R, G)=n-n / p$ where $p$ is the minimal odd prime factor of $n$. The assertion follows. 
(3) As before, let $R^{\prime}$ be the image of $R$ in $B / I$ and let $T$ be the subalgebra of $R^{\prime}$ generated by $\left\{x_{i}^{2}\right\}_{i=1}^{n}$. Then $T$ is commutative and GKdim $T=\operatorname{GKdim} R^{\prime}=$ GKdim $B / I$. It suffice to show that

$$
\mathrm{GKdim} T / \mathfrak{p} \leq n- \begin{cases}\phi(n), & 4 \nmid n, \\ \frac{\phi(n)}{2}, & 4 \mid n,\end{cases}
$$

for all minimal prime $\mathfrak{p}$ of $T$.

Let $\xi$ be a primitive $n$th root of unity. For any $0 \leq i \leq n-1$, let $y_{i}=\sum_{j=1}^{n} \xi^{i j} x_{j}$ and $Y_{i}=\sum_{j=1}^{n} \xi^{i j} x_{j}^{2}$. Since $x_{i} x_{j}+x_{j} x_{i}=0$ for all $i \neq j$, we have $y_{i}^{2}=Y_{2 i}$. Since $x_{i}^{2}$ are central in $R$ for all $i, Y_{i}$ are all central in $R$ for all $i$. Note that the commutative $\operatorname{ring} T / \mathfrak{p}$ is generated by $\left\{x_{i}^{2}\right\}_{i=1}^{n}$, hence by $\left\{Y_{i}\right\}_{i=1}^{n}$.

Let $N_{n}$ denote the cardinality of $\left\{Y_{2 i} \mid \operatorname{gcd}(i, n)=1, i=0,1, \cdots, n-1\right\}$. It is easily checked that $N_{n}$ is $\phi(n)$ when $4 \nmid n$; and $\frac{1}{2} \phi(n)$ when $4 \mid n$. We give some details in the case when $n=2 m$ where $m>1$ is an odd integer. It suffices to show that $Y_{2 i_{0}} \neq Y_{2 i_{1}}$ if $\operatorname{gcd}\left(i_{0}, n\right)=\operatorname{gcd}\left(i_{1}, n\right)=1$ and $0 \leq i_{0}<i_{1} \leq n-1$. If $Y_{2 i_{0}}=Y_{2 i_{1}}$, then $n \mid 2\left(i_{1}-i_{0}\right)$. This implies that $m \mid\left(i_{1}-i_{0}\right)$. Since both $i_{1}$ and $i_{0}$ are odd $\left(\operatorname{asgcd}\left(i_{0}, n\right)=\operatorname{gcd}\left(i_{1}, n\right)=1\right), 2 m \mid\left(i_{1}-i_{0}\right)$, which is impossible. So $N_{n}=\phi(n)$ in this case.

Suppose $\operatorname{gcd}\left(n, i_{0}\right)=1$. By Lemma 5.1 $y_{i_{0}}^{n}=0$ in $R^{\prime}$ (by taking $f_{i}=y_{i_{0}}$ for all $i$ ). Then $Y_{2 i_{0}}^{n}=y_{i_{0}}^{2 n}=0$. Since $T / \mathfrak{p}$ is prime, $Y_{2 i_{0}}=0$ in $T / \mathfrak{p}$. Therefore $T / \mathfrak{p}$ is generated by no more than $\left(n-N_{n}\right)$ elements. This implies that $\operatorname{GKdim} T / \mathfrak{p} \leq$ $n-N_{n}$ and the assertion follows.

(4) This is an immediate consequence of parts $(1,2)$.

Now we are ready to prove Theorem 0.5 .

Proof of Theorem 0.5. Note that $G=W$. By Theorem $5.7(1,3), \mathrm{p}(R, W) \geq 2$. It is well-known that skew polynomial rings are CM. As $G$ is a subgroup of $A u t_{g r}(R)$, the $\mathbb{k} G$-action on $R$ is clearly inner faithful. Then the final assertion follows from Theorem 0.3 .

Let $S_{n}$ act on $\left\{x_{1}, \cdots, x_{n}\right\}$ as permutations. This action extends to an algebraic action on both commutative polynomial ring $\mathbb{k}\left[x_{1}, \cdots, x_{n}\right]$ and the skew polynomial ring $\mathbb{k}_{-1}\left[x_{1}, \cdots, x_{n}\right]$. We define the pertinency for $S_{n}$ and its subgroups in these cases.

Definition 5.8. Suppose char $\mathbb{k}=0$. Let $G$ be a subgroup of $S_{n}$ that acts naturally on $\mathbb{k}\left[x_{1}, \cdots, x_{n}\right]$ and $\mathbb{k}_{-1}\left[x_{1}, \cdots, x_{n}\right]$ respectively. Define the positive and negative pertinency of $G$ to be

$$
\mathrm{p}_{+}(G)=\mathrm{p}\left(\mathbb{k}\left[x_{1}, \cdots, x_{n}\right], G\right) \quad \text { and } \quad \mathrm{p}_{-}(G)=\mathrm{p}\left(\mathbb{k}_{-1}\left[x_{1}, \cdots, x_{n}\right], G\right)
$$

respectively.

Question 5.9. Is there a combinatorial algorithm to compute $\mathrm{p}_{+}(G)$ and $\mathrm{p}_{-}(G)$ for all $G \subseteq S_{n}$ ?

The second part of the above question concerning $\mathrm{p}_{-}(G)$ was suggested by Ellen Kirkman. See [BHZ, Section 7] for more comments. 
Acknowledgments. The authors thank the referee for a very careful reading and useful comments. Many thanks to Kenneth Chan, Andrew Conner, Jason Gaddis, Frank Moore, Fredy Van Oystaeyen, Chelsea Walton, Yinhuo Zhang for sharing their (un-published) notes and interesting ideas; special thanks to Ellen Kirkman for many useful conversations on the subject, for suggesting a question which leads to Theorem 0.5 and Question 5.9. for sharing her ideas concerning Lemma 3.10, and for pointing out a mistake of Theorem 5.7 in an earlier version. Both Y.-H. Bao and J.-W. He were supported by NSFC (No. 11571239, 11401001,11671351) and J.J. Zhang by the US National Science Foundation (grant Nos. DMS 1402863 and DMS 1700825).

\section{REFERENCES}

[ASZ1] K. Ajitabh, S.P. Smith, and J.J. Zhang, Auslander-Gorenstein rings, Comm. Algebra 26 (7) (1998), 2159-2180.

[ASZ2] K. Ajitabh, S.P. Smith, and J.J. Zhang, Injective resolutions of some regular rings, J. Pure Appl. Algebra 140 (1) (1999), 1-21.

[ATV] M. Artin, J. Tate, M. Van den Bergh, Modules over Regular Algebras of Dimension 3, Invent. Math. 106 (1991), 335-389.

[AZ] M. Artin, J.J. Zhang, Noncommutative projective schemes, Adv. Math. 109 (1994), 228-287.

[Au1] M. Auslander, On the purity of the branch locus, Amer. J. Math. 84 (1962), 116-125.

[Au2] M. Auslander, Rational singularities and almost split sequences, Trans. Amer. Math. Soc. 293 (1986), 511-531.

[AR] M. Auslander and I. Reiten, $k$-Gorenstein algebras and syzygy modules, J. Pure Appl. algebra 92 (1994), 1-27.

[BHZ] Y.-H. Bao, J.-W. He and J.J. Zhang, Noncommutative Auslander theorem, Trans. Amer. Math. Soc. (accepted for publication), preprint (2016), arXiv:1607.06955

$[\mathrm{BCF}]$ J. Bergen, M. Cohen and D. Fischman, Irreducible actions and faithful actions of Hopf algebras, Israel J. Math. 72 (1990), 5-18.

[BGG] I.N. Bernstein, I.M. Gelfand and S. I. Gelfand, Algebraic bundles over $p$ and problems in linear algebra, Funct. Anal. Appl. 12 (1979), 212-214.

[BGS] A. Beilinson, V. Ginzburg and W. Soergel, Koszul duality patterns in representation theory, J. Amer. Math. Soc. 9 (1996), 473-527.

[Be] A. Beligiannis, The homological theory of contravariantly finite subcategories: AuslanderBuchweitz contexts, Gorenstein categories and (co-)stabilization, Comm. Algebra 28 (2000), $4547-4596$.

[Bu] R.-O., Buchweitz, From Platonic Solids to Preprojective Algebras via the McKay Correspondence Oberwolfach Jahresbericht Annual Report 2012, 18-28.

[Ca] J.F. Carlson, The decomposition of the trivial module in the complexity quotient category, J. Pure Appl. Algebra 106 (1996), 23-44.

[CDW] J.F. Carlson, P. W. Donovan and W. W. Wheeler, Complexity and quotient categories for group algebras, J. Pure Appl. Algebra 93 (1994), 147-167.

[Ch] D. Chan, McKay correspondence for canonical orders, Trans. Amer. Math. Soc. 362 (4) (2010), 1765-1795.

[CKWZ1] K. Chan, E. Kirkman, C. Walton and J.J. Zhang, Quantum binary polyhedral groups and their actions on quantum planes, J. Reine Angew. Math. 719 (2016), 211-252.

[CKWZ2] K. Chan, E. Kirkman, C. Walton and J.J. Zhang, McKay Correspondence for semisimple Hopf actions on regular graded algebras I, arXiv:1607.06977

[CKWZ3] K. Chan, E. Kirkman, C. Walton and J.J. Zhang, McKay Correspondence for semisimple Hopf actions on regular graded algebras II, arXiv:1610.01220

[GW] K.R. Goodearl, R.B. Warfield, Jr., An introduction to noncommutative noetherian rings (second edition), London Math. Soc. Student Texts 61, Cambridge University Press, 2004.

[GM] E.L. Green, R. Martínez-Villa, Koszul algebra Yoneda algebras II, CMS Conference Proc. 24 (1998), 227-244.

[GLW] J.-Y. Guo, A. Li, Q. Wu, Selfinjective Koszul algebras of finite complexity, Acta Math. Sinica, English Series 25 (2009), 2179-2198. 
[HVZ1] J.-W. He, F. Van Oystaeyen and Y. Zhang, Hopf algebra actions on differential graded algebras and applications, Bull. Belg. Math. Soc. Simon Stevin 18 (2011), 99-111.

[HVZ2] J.-W. He, F. Van Oystaeyen and Y. Zhang, Hopf dense Galois extensions with applications, J. Algebra 476 (2017), 134-160.

[HW] J.-W. He and Q.-S. Wu, Koszul differential graded algebras and BGG correspondence II, Chin. Ann. Math. 31 B(1) (2010), 133-144.

[IT] O. Iyama and R. Takahashi, Tilting and cluster tilting for quotient singularities, Math. Ann. 356 (2013), 1065-1105.

[Jo1] P. Jørgensen, Linear free resolutions over non-commutative algebras, Compositio Math. 140 (2004), 1053-1058.

[Jo2] P. Jørgensen, A noncommutative BGG correspondence, Pacific J. Math. 218 (2005), 357378.

[Ki] E. Kirkman, Private communication, (2015).

[KKZ1] E. Kirkman, J. Kuzmanovich and J.J. Zhang, Gorenstein subrings of invariants under Hopf algebra actions, J. Algebra 322 (2009), 3640-3669.

[KKZ2] E. Kirkman, J. Kuzmanovich and J.J. Zhang, Invariants of (-1)-skew polynomial rings under permutation representations, Recent advances in representation theory, quantum groups, algebraic geometry, and related topics, 155-192, Contemp. Math., 623, Amer. Math. Soc., Providence, RI, 2014.

[KKZ3] E. Kirkman, J. Kuzmanovich and J.J. Zhang, Rigidity of graded regular algebras, Trans. Amer. Math. Soc. 360 (12) (2008), 6331-6369.

[KKZ4] E. Kirkman, J. Kuzmanovich and J.J. Zhang, Noncommutative complete intersections, J. Algebra 429 (2015), 253-286.

[KL] G.R. Krause and T.H. Lenagan, Growth of algebras and Gelfand-Kirillov dimension, Research Notes in Mathematics, Pitman Adv. Publ. Program, 116 (1985).

[Le1] G.J. Leuschke, The McKay Correspondence, http://www.leuschke.org/uploads/McKay-total.pdf

[Le2] G.J. Leuschke, Non-commutative crepant resolutions: scenes from categorical geometry, Progress in commutative algebra 1, 293-361, de Gruyter, Berlin, 2012.

[Ma] R. Martinéz-Villa, Graded, selfinjective, and Koszul algebras, J. Algebra 215 (1) (1999), $34-72$.

[MR] J.C. McConnell and J.C . Robson, "Noncommutative Noetherian Rings," Wiley, Chichester, 1987.

[MM] H. Minamoto and I. Mori, The structure of AS-Gorenstein algebras, Adv. Math. 226 (2011), 4061-4095.

[Mi] J.I. Miyachi, Localization of triangulated categories and derived categories, J. Algebra 141 (1991), 463-483.

[Mon] S. Montgomery, Hopf Algebras and Their Actions on Rings, CBMS Reg. Conf. Ser. Math. 82, Amer. Math. Soc., Providence, RI, 1993.

[Mor1] I. Mori, Riemann-Roch like theorem for triangulated categories, J. Appl. Algebra, 193 (2004), 263-285.

[Mor2] I. Mori, McKay-type correspondence for AS-regular algebras, J. Lond. Math. Soc. (2) 88 (2013), no. 1, 97-117.

[MU] I. Mori and K. Ueyama, Ample Group Action on AS-regular Algebras and Noncommutative Graded Isolated Singularities, Trans. Amer. Math. Soc., 368 (2016), no. 10, 7359-7383

[Ne] C. Negron, Spectral sequences for the cohomology rings of a smash product, J. Algebra 433 (2015), 73-106.

[Or] D. Orlov, Derived categories of coherent sheaves and triangulated categories of singularities, in: Algebra, Arithmetic, and Geometry, Progress Math. 270 (2009), 503-531.

[Pr] S.B. Priddy, Koszul resolutions, Trans. Amer. Math. Soc. 152 (1970), 39-60.

[RRZ] M. Reyes, D. Rogalski and J.J. Zhang, Skew Calabi-Yau algebras and homological identities, Adv. Math. 264 (2014), 308-354.

[Ro] J.J. Rotman, "An introduction to homological algebra" Pure and Applied Mathematics 85, Academic Press, Inc. New York-London, 1979.

[SkV] S. Skryabin and F. Van Oystaeyen, The Goldie Theorem for H-semiprime algebras, J. Algebra, 305 (2006), 292-320.

[SmV] S.P. Smith, M. Van den Bergh, Noncommutative quadric surfaces, J. Noncomm. Geometry 7 (2013), 817-856. 
[SZ] J.T. Stafford and J.J. Zhang, Homological properties of (graded) Noetherian PI rings, J. Algebra 168 (3) (1994), 988-1026.

[StZ] D.R. Stephenson, J.J. Zhang, Growth of graded Noetherian rings, Proc. Amer. Math. Soc. 125 (1997), 1593-1605.

[St] D. Stefan, Hochschild cohomology on Hopf galois extensions, J. Pure Appl. Algebra 103 (2) (1995), 221-233.

[Sw] M. E. Sweedler, Integrals for Hopf algebras, Ann. Math. 89 (1969), 323-335.

[Ue] K. Ueyama, Graded maximal Cohen-Macaulay modules over noncommutative graded Gorenstein isolated singularities, J. Algebra 383 (2013), 85-103.

[We] C.A. Weibel, An introduction to homological algebra, volume 38. Cambridge university press, 1995.

[Wem] M. Wemyss, The GL(2, C) McKay correspondence, Math. Ann. 350 (3) (2011), 631-659.

[YZ] A. Yekutieli and J.J. Zhang, Rings with Auslander dualizing complexes, J. Algebra 213 (1) (1999), 1-51.

[Yo] Y. Yoshino, Cohen-Macaulay Modules over Cohen-Macaulay Rings, London Math. Soc. Lecture Note Series 146, Cambridge University Press, Cambridge, 1990.

[Zh] J.J. Zhang, Connected graded Gorenstein algebras with enough normal elements, J. Algebra 189 (2), (1997), 390-405.

Bao: School of Mathematical Sciences, Anhui University, Hefei, Anhui, 230601, China

E-mail address: baoyh@ahu.edu.cn, yhbao@ustc.edu.cn

He: Department of Mathematics, Hangzhou Normal University, Hangzhou Zhejiang 310036, CHINA

E-mail address: jwhe@hznu.edu.cn

Zhang: Department of Mathematics, Box 354350, University of Washington, Seattle, WASHINGTON 98195, USA

E-mail address: zhang@math.washington.edu 